\title{
An Analogue of the Nearest Integer Continued Fraction for Certain Cubic Irrationalities
}

\author{
By H. C. Williams* and G. W. Dueck
}

\begin{abstract}
Let $\theta$ be any irrational and define $N e(\theta)$ to be that integer such that $|\theta-N e(\theta)|<\frac{1}{2}$. Put $\rho_{0}=\theta, r_{0}=N e\left(\rho_{0}\right), \rho_{k+1}=1 /\left(r_{k}-\rho_{k}\right), r_{k+1}=N e\left(\rho_{k+1}\right)$. Then the $r$ 's here are the partial quotients of the nearest integer continued fraction (NICF) expansion of $\theta$. When $D$ is a positive nonsquare integer, and $\theta=\sqrt{D}$, this expansion is periodic. It can be used to find the regulator of $\mathcal{L}(\sqrt{D})$ in less than 75 percent of the time needed by the usual continued fraction algorithm. A geometric interpretation of this algorithm is given and this is used to extend the NICF to a nearest integer analogue of the Voronoi Continued Fraction, which is used to find the regulator of a cubic field $\mathscr{F}$ with negative discriminant $\Delta$. This new algorithm (NIVCF) is periodic and can be used to find the regulator of $\mathscr{F}$.

If $I<\sqrt[4]{|\Delta| / 148}$, the NIVCF algorithm can be used to find any algebraic integer $\alpha$ of $\mathscr{F}$ such that $N(\alpha)=I$. Numerical results suggest that the NIVCF algorithm finds the regulator of $\mathscr{F}=\mathcal{Q}(\sqrt[3]{D})$ in about 80 percent of the time needed by Voronoi's algorithm.
\end{abstract}

1. Introduction. Let $\theta$ be any real number. We find the Regular Continued Fraction (RCF) expansion of $\theta$ by putting $\phi_{0}=\theta, q_{0}=\left[\phi_{0}\right], \phi_{i+1}=1 /\left(\phi_{i}-q_{i}\right), q_{i+1}=\left[\theta_{i+1}\right]$ $(i=0,1,2, \ldots, n, \ldots)$ and denote this as

$$
\theta=\left[q_{0}, q_{1}, q_{2}, \ldots, q_{n-1}, \phi_{n}\right] .
$$

Here $[\alpha]$ denotes that integer such that $\alpha-1<[\alpha] \leqslant \alpha$. We also define the convergent $C_{n}=\left[q_{0}, q_{1}, q_{2}, \ldots, q_{n}\right]$. Further, if $A_{-2}=0, A_{-1}=1, B_{-2}=0, B_{-1}=1$,

$$
A_{k+1}=q_{k+1} A_{k}+A_{k-1}, \quad B_{k+1}=q_{k+1} B_{k}+B_{k-1} \quad(k=-1,0,1,2, \ldots),
$$

then $C_{n}=A_{n} / B_{n}$.

The Nearest Integer Continued Fraction (NICF) of $\theta$ is found by setting $\rho_{0}=\theta$, $r_{0}=N e\left(\rho_{0}\right)$ and calculating $\rho_{i+1}=1 /\left(r_{i}-\rho_{i}\right), r_{i+1}=N e\left(\rho_{i+1}\right)(i=0,1,2, \ldots$, $n, \ldots)$. We denote this by

$$
\theta=\left(r_{0}, r_{1}, r_{2}, \ldots, r_{n-1}, \rho_{n}\right)
$$

Here $N e(\alpha)$ is that integer such that $|\alpha-N e(\alpha)|<\frac{1}{2}$; that is, $N e(\alpha)$ is the nearest integer to $\alpha$. The convergent $\bar{C}_{n}=\left(r_{0}, r_{1}, r_{2}, \ldots, r_{n}\right)$ can be calculated by noting that $\bar{C}_{n}=\bar{A}_{n} / \bar{B}_{n}, \bar{A}_{-2}=0, \bar{A}_{-1}=1, \bar{B}_{-2}=-1, \bar{B}_{-1}=0$, and

$$
\overline{A_{k+1}}=r_{k+1} \overline{A_{k}}-\overline{A_{k-1}}, \quad \bar{B}_{k+1}=r_{k+1} \bar{B}_{k}-\bar{B}_{k-1} \quad(k=-1,0,1,2,3, \ldots) .
$$

Received March 8, 1983.

1980 Mathematics Subject Classification. Primary 10F20, 10A32, 12A30.

* Research supported by NSERC of Canada grant number A7649 and the I. W. Killam Program. 
The NICF was introduced in 1873 by Minnegerode [4] who used the NICF expansion of $\sqrt{D}$ to solve the Pell Equation

$$
t^{2}-D u^{2}=1
$$

Later Hurwitz [3] gave further results concerning the NICF and more recently other results have been obtained by Williams [7], Williams and Buhr [6], and Adams [1].

For example, in [6], the following theorem is proved.

THEOREM 1.1. If $c=(3+\sqrt{5}) / 2$ and $a, b$ are coprime integers such that

$$
|a / b-\theta|<1 / c b^{2}
$$

for some irrational $\theta$, then $a / b$ must be a convergent in the NICF expansion of $\theta$.

In this paper we shall at first be concerned with NICF expansions of quadratic irrationals. To this end we let $D$ be a square free positive integer and put $\mathscr{K}=2 \cdot(\sqrt{D})$, the quadratic field formed by adjoining $D$ to the rationals 2 . Let $\theta_{\mathcal{K}}$ be the ring of algebraic integers in $\mathscr{K}$. $\Theta_{\mathscr{K}}$ has as basis $\{1, \mu\}$, where $\mu=(1+\sqrt{D}) / 2$ when $D \equiv 1$ $(\bmod 4)$ and $\mu=\sqrt{D}$ otherwise. Let $\varepsilon_{0}(>1)$ be the fundamental unit of $\mathcal{K}$ and let $\Delta$ be the discriminant of $\mathcal{K}$. If $\alpha \in \mathcal{K}$, let $\alpha^{\prime}$ denote the conjugate of $\alpha$ and $N(\alpha)=\alpha \alpha^{\prime}$ be the norm of $\alpha$.

It is well known that if $\theta \in \mathcal{K}$, the RCF expansion of $\theta$ is periodic. In fact, if $\theta=\mu$, then

$$
\mu=\left[q_{0}, \overline{q_{1}, q_{2}, \ldots, q_{p}}\right],
$$

where we draw a bar over the periodic part of the continued fraction. From results in [3], we find that if $\theta=\mu$, then the NICF expansion of $\theta$ is also periodic; further,

$$
\mu=\left(r_{0}, \overline{r_{1}, r_{2}, \ldots, r_{\pi}}\right)
$$

when $N\left(\varepsilon_{0}\right)=1$ and

$$
\mu=\left(r_{0}, \overline{r_{1}, r_{2}, \ldots, r_{\pi},-r_{1},-r_{2}, \ldots,-r_{\pi}}\right)
$$

when $N\left(\varepsilon_{0}\right)=-1$. In fact we have the following theorem.

THEOREM 1.2. If $D \neq 5$, then $\varepsilon_{0}=\left|\overline{A_{\pi-1}}-\mu^{\prime} \bar{B}_{\pi-1}\right|$; if $D=5$, then $\varepsilon_{0}^{2}=\mid \overline{A_{\pi-1}}-$ $\mu^{\prime} \bar{B}_{\pi-1} \mid$.

Of course this result shows us how to solve (1.1) by using the NICF expansion of $\mu$.

Theorem 1.2 can be regarded as a special case of

THEOREM 1.3. If $\alpha=x+y \mu$, where $x, y$ are coprime rational integers, $\alpha>1$ and $|N(\alpha)|<\sqrt{\Delta / c_{1}}$, where $c_{1}=c^{2}=(7+3 \sqrt{5}) / 2 \simeq 6.85$, then

$$
\alpha=\left|\overline{A_{m}}-\mu^{\prime} \bar{B}_{m}\right|
$$

for some $m \geqslant 1$.

Proof. This result can be derived by using Theorem 1.1 and the simple observation that if $\bar{C}_{n}(n \geqslant 0)$ is any convergent in the NICF expansion of $\theta(>2)$, then $-\left(\bar{C}_{n}\right)^{-1}$ is a convergent in the NICF expansion of $-1 / \theta$.

Thus, the NICF can be used to find all elements in $\theta_{\mathcal{K}}$ with norm bounded by $\sqrt{\Delta / c_{1}}$. For, if $N(\alpha)=N$ and $|N|<\sqrt{\Delta / c_{1}}$ and $\alpha<1$, then $\beta= \pm \varepsilon_{0}^{k} \alpha>1$ for some 
$k$ and $|N(\beta)|=|N(\alpha)|$. Since $\varepsilon_{0}$ and $\beta$ can be found from the NICF expansion of $\mu$, so can $\alpha$. Further, if $\alpha=x+y \sqrt{D}$ and $\operatorname{gcd}(x, y)=d>1$, then, since $\left|N / d^{2}\right|<|N|$, we can find $\alpha / d$ from the NICF expansion of $\mu$.

From the several numerical results presented in [6] it seems that the ratio $\pi / p$ is usually about .69. In fact Adams [1] gives results which suggest that this ratio should be $\log ((1+\sqrt{5}) / 2) / \log 2$ in the limit. This, of course, means that we can usually calculate $\varepsilon_{0}$ more rapidly by using the NIVCF algorithm rather than the NICF algorithm. Also, if we are attempting to solve the Diophantine equation

$$
N(\alpha)=I \text {, }
$$

where $\alpha \in \theta_{\mathscr{K}}$ and $|I|<\sqrt{\Delta / c_{1}}$, we should use the NICF algorithm rather than the RCF.

Let $\delta$ be the real zero of an irreducible (over 2) cubic polynomial with integer coefficients and negative discriminant. In order to find the fundamental unit $\varepsilon_{0}$ $(>1)$ in $\mathcal{F}=2(\delta)$, Voronoi's Continued Fraction (VCF) algorithm is often used. (See Delone and Faddeev [2] or Williams, Cormack, Seah [8].) This algorithm, which is particularly suited for the problem of finding the fundamental unit(s) in a cubic field, is an extension of the RCF algorithm. In view of the increased speed obtained by using the NICF algorithm over the RCF, it would be desirable to develop an analogue of the NICF algorithm for cubic irrationalities. In this paper we shall develop such an algorithm, called the nearest integer analogue of the Voronoi Continued Fraction (NIVCF), for use with elements from $\mathscr{F}$ above. We will also prove theorems analogous to Theorems 1.2 and 1.3 and compare (numerically) the speed of the NIVCF algorithm with that of the VCF. In order to do all of this we must first place the RCF and NICF in a geometric setting similar to that used for Voronoi's algorithm in [2] and [8]. This will be done in the next two sections.

2. The Lattices $\delta$ and $R$. In this section we give a very brief description of certain properties of some special lattices $\delta$ and $\Re$. While we confine our discussion here to these special lattices, it should be pointed out that much of what is said here can be extended to more general lattices. See, for example, the approach of Voronoi [5] and the general results given in Delone and Faddeev [2].

Let $\mathcal{E}_{n}$ represent Euclidean $n$-space. If $\beta \in \mathscr{K}$, the point $B$ of $\mathscr{E}_{2}$ corresponding to $\beta$ is $\left(\beta, \beta^{\prime}\right)$. If $\lambda, \mu \in \mathcal{K}$, and $\lambda, \mu$ are rationally independent, define lattice $\delta$ by

$$
\delta=\{u \Lambda+v M \mid u, v \in \mathbf{Z}\},
$$

where $\Lambda=\left(\lambda, \lambda^{\prime}\right), M=\left(\mu, \mu^{\prime}\right)$. We say that $\delta$ is the lattice with basis $\{\lambda, \mu\}$ or $\{\Lambda, M\}$ and will denote it by $\langle\lambda, \mu\rangle$.

If $\alpha \in \mathscr{F}$, let $\alpha^{\prime}, \alpha^{\prime \prime}$ denote the conjugates of $\alpha$ and define $A$, the point of $\mathcal{E}_{3}$ corresponding to $\alpha$, to be

$$
A=\left(\alpha,\left(\alpha^{\prime}-\alpha^{\prime \prime}\right) / 2 i,\left(\alpha^{\prime}+\alpha^{\prime \prime}\right) / 2\right),
$$

where $i^{2}+1=0$. Since the discriminant of the polynomial of which $\alpha$ is a zero is negative, we see that $A$ is a point with real coordinates. If $\lambda, \mu, \nu \in \mathscr{F}$ and $\lambda, \mu, \nu$ are rationally independent, define the lattice $R$ by

$$
\mathscr{F}=\{u \Lambda+v M+w N \mid u, v, w \in \mathbf{Z}\} .
$$

Here $\Re$ is the lattice with basis $\{\lambda, \mu, \nu\}$ and will be denoted by $\langle\lambda, \mu, \nu\rangle$. 


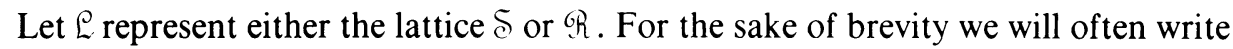
$\alpha \in \mathcal{L}$ as a means of denoting that it is the corresponding point $A$ of $\varepsilon_{2}$ or $\mathcal{E}_{3}$ that is actually in $\mathcal{L}$. Also, if $\mathcal{L}=\langle\lambda, \mu\rangle$ and $\alpha \in \mathscr{K}$ we define $\alpha \mathcal{L}$ to be $\langle\alpha \lambda, \alpha \mu\rangle$; similarly, if $\mathcal{L}=\langle\lambda, \mu, \nu\rangle$ and $\alpha \in \widetilde{\mathscr{y}}$, we define $\alpha \mathscr{L}$ to be $\langle\alpha \lambda, \alpha \mu, \alpha \nu\rangle$. Note that under this convention, if $\alpha \varrho_{1}=\alpha \varrho_{2}$ and $\alpha \neq 0$, then $\varrho_{1}=\varrho_{2}$.

If $A=\left(\alpha, \alpha^{\prime}\right)$ is a point of $\S$, we define the normed body of $A, \mathfrak{N}(A)$ or $\Re(\alpha)$, to be

$$
\mathfrak{X}(A)=\mathfrak{X}(\alpha)=\left\{(x, y)\left|(x, y) \in \mathcal{E}_{2},\right| x|<| \alpha|,| y|\leqslant| \alpha^{\prime} \mid\right\} \text {. }
$$

This is a semiopen rectangle which is symmetric about the origin $O$ of $\varepsilon_{2}$. If $A$ is a point of $\mathcal{R}$, we define the normed body of $A$ to be

$$
\Re(A)=\mathscr{T}(\alpha)=\left\{\left.(x, y, z)\left|(x, y, z) \in \varepsilon_{3},\right| x|<| \alpha\left|, y^{2}+z^{2} \leqslant\right| \alpha^{\prime}\right|^{2}\right\} .
$$

This is a semiopen right circular cylinder, symmetric about the origin $O$ of $\varepsilon_{3}$ with axis the $x$-axis of $\varepsilon_{3}$. We point out here that if $\left|\alpha^{\prime}\right|=\left|\beta^{\prime}\right|$, then $\alpha= \pm \beta$ (see [2, p. 274]). Thus, if $\left|\beta^{\prime}\right|=\left|\alpha^{\prime}\right|$, then $B \notin \mathcal{N}(\alpha)$.

We say that $\phi(\neq 0)$ or the point $\Phi$ corresponding to $\phi$ is a minimum of $\varrho$ if $\mathcal{X}(\phi) \cap \mathcal{L}=\{O\}$. If $\psi$ and $\phi$ are minima of $\mathcal{L}$ and $\psi>\phi$, we say that $\psi$ and $\phi$ are adjacent minima when there does not exist a nonzero $\theta \in \mathcal{E}$ such that $\phi<\theta<\psi$ and $\left|\theta^{\prime}\right|<\left|\phi^{\prime}\right|$. If

$$
\theta_{1}, \theta_{2}, \theta_{3}, \ldots, \theta_{n}, \ldots
$$

is a sequence of minima of $\varrho$ such that $\theta_{i+1}>\theta_{i}$ and $\theta_{i+1}, \theta_{i}$ are adjacent, we call

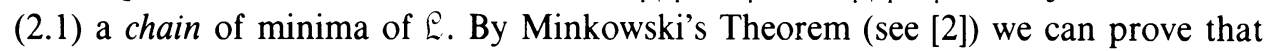
there always exist such chains in $\varrho$. Further, if $\theta$ is any minimum of $\varrho$ and $\theta>\theta_{1}$, then $\theta=\theta_{m}$ for some integer $m>1$.

If $\mathcal{E}=\langle 1, \mu\rangle$ or $\mathcal{E}=\langle 1, \mu, \nu\rangle$ and $\varrho$ has 1 as a minimum, we say that $E$ is a reduced lattice. We now give some theorems concerning these reduced lattices.

THEOREM 2.1. Let $\varrho$ be a reduced lattice and let $\theta(>1)$ be the minimum of $\subseteq$ adjacent to 1 . There exists a basis of $E$ which includes 1 and $\theta$.

Proof. The proof of this result for $\mathcal{E}=\langle 1, \mu, \nu\rangle$ is given in Section 2 of [10]. The proof for $\mathcal{E}=\langle 1, \mu\rangle$ is similar but easier.

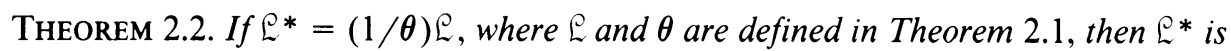
a reduced lattice.

Proof. By Theorem 2.1 we see that $\varrho^{*}$ has a basis which includes 1 . If $\varrho^{*}$ is not reduced, there must exist $\alpha \in \mathcal{L}^{*}$ such that $|\alpha|<1,\left|\alpha^{\prime}\right|<1$, and $\alpha \neq 0$. Since $\alpha \theta \in \mathcal{L}$, it follows that $|\alpha \theta|<|\theta|,\left|\alpha^{\prime} \theta^{\prime}\right|<\left|\theta^{\prime}\right|$ and $\alpha \theta \neq 0$. This contradicts the definition of $\theta$.

TheOREM 2.3. Let $\mathcal{L}^{*}, \mathcal{L}, \theta$ be as defined in Theorem 2.2 and let $\omega(>\theta)$ be the minimum adjacent to $\theta$ in $\mathcal{L}$. If $\theta^{*}(>1)$ is the minimum adjacent to 1 in $\mathrm{L}^{*}$, then $\omega=\theta \theta^{*}$.

Proof. Let $\phi=\theta \theta^{*}$. We have $\phi>\theta$ and $\left|\phi^{\prime}\right|<\left|\theta^{\prime}\right|$; thus, if $\phi \neq \omega$, then $\omega<\theta \theta^{*}$, and $\left|\omega^{\prime}\right|<\left|\theta^{\prime}\right|$. Since $\omega / \theta \neq 0, \omega / \theta \in \mathcal{L}^{*}, 0<\omega / \theta<\theta^{*}$, and $\left|\omega^{\prime} / \theta^{\prime}\right|<1$, we have a contradiction to the definition of $\theta^{*}$; thus, $\omega=\phi$. 
COROLlary. If $\Re=(1 / \omega) \mathcal{L}$, then $\Re$ is a reduced lattice.

Let $\mathcal{L}_{1}=\mathcal{E}$ be any reduced lattice. Let $\theta_{g}^{(n)}(>1)$ be the minimum in $\mathcal{L}_{n}$ adjacent to 1 and define $\mathscr{L}_{n+1}=\left(1 / \theta_{g}^{(n)}\right) \mathscr{L}_{n}$. By the theorems above we see that each of the $\mathscr{L}_{i}$ is a reduced lattice. Further, if $\theta_{1}$ in (2.1) is 1 , then

$$
\theta_{n}=\prod_{i=1}^{n-1} \theta_{g}^{(i)}
$$

and

$$
\theta_{n} e_{n}=e_{1} .
$$

If $\mathcal{L}$ has as its basis a basis of $\theta_{\mathscr{K}}$ or $\theta_{\mathscr{F}}$, then $\mathcal{E}$ is reduced and $\varepsilon_{0} \in \mathcal{L}$. Also, since $\left|N\left(\varepsilon_{0}\right)\right|=1, \varepsilon_{0}$ must be a minimum of $\mathcal{L}$; consequently, when $\theta_{1}=1$ we have $\varepsilon_{0}=\theta_{p+1}$ for some $p \geqslant 1$. Since $\mathscr{L}_{1}=\varepsilon_{0} \mathscr{L}_{p+1}=\mathscr{L}_{p+1}$, we see that the algorithm for finding the $\mathcal{L}_{i}$ is periodic with period length $p$.

We conclude this section with the following simple result.

LEMMA 2.4. If $\theta(>1)$ is the minimum adjacent to 1 in a reduced lattice $R=\langle 1, \mu, \nu\rangle$, then $\left|\theta^{\prime}-1\right|>1$.

Proof. Suppose $\left|\theta^{\prime}-1\right| \leqslant 1$. If we put $\phi=\theta-1 \in \Re$, then $0<\phi<\theta$ and $\left|\phi^{\prime}\right| \leqslant 1$. If $\left|\phi^{\prime}\right|=1$, then $\phi= \pm 1$ and $\theta=2$, which is not possible. If $\left|\phi^{\prime}\right|<1$ we have a contradiction to the defintion of $\theta$; hence, $\left|\theta^{\prime}-1\right|>1$.

3. The RCF and NICF in $\delta$. Let $\ell_{1}=\delta_{1}=\left\langle 1, \mu_{1}\right\rangle$ be a reduced lattice. From the results of Section 2 we see that the elements in a chain (2.1) in $\delta_{1}$ can be found from (2.2) once we know how to find the minimum $\theta(>1)$ adjacent to 1 in any reduced lattice $\delta=\langle 1, \mu\rangle$. The following theorem of Voronoi [5] can be used to do this.

THeorem 3.1. Let $\delta=\langle\theta, \psi\rangle$ and suppose that $\psi>\theta>0$. Then $\theta$ and $\psi$ are adjacent minima of $S$ if and only if $\left|\theta^{\prime}\right|>\left|\psi^{\prime}\right|$ and $\psi^{\prime} \theta^{\prime}<0$.

Proof. If $\psi$ and $\theta$ are adjacent minima of $\delta$, then by definition we have $\left|\theta^{\prime}\right|>\left|\psi^{\prime}\right|$. Since $0<\psi-\theta<\psi$, we must have $\left|\psi^{\prime}-\theta^{\prime}\right|>\left|\psi^{\prime}\right|$; otherwise, $\psi$ could not be a minimum. Hence $\theta^{\prime} \psi^{\prime}<0$.

Suppose $\left|\theta^{\prime}\right|>\left|\psi^{\prime}\right|$ and $\theta^{\prime} \psi^{\prime}<0$. If $\theta$ is not a minimum of $\delta$, there must exist $\gamma \in \mathcal{S}$ such that $|\gamma|<\theta$ and $\left|\gamma^{\prime}\right|<\left|\theta^{\prime}\right|$. Since $\gamma=a \theta+b \psi(a, b \in \mathbf{Z})$, this means that

$$
|a \theta+b \psi|<\theta, \quad\left|a \theta^{\prime}+b \psi^{\prime}\right|<\left|\theta^{\prime}\right| .
$$

Clearly, neither $a$ nor $b$ is zero; but, if $a b>0$, then $|a \theta+b \psi|>\theta$ and if $a b<0$, then $\left|a \theta^{\prime}+b \psi^{\prime}\right|>\left|\theta^{\prime}\right|$. Thus, $\theta$ is a minimum of $\delta$ and by similar reasoning so is $\psi$. Further, there cannot exist $a, b \in \mathbf{Z}$ such that both $|a \theta+b \psi|<\psi$ and $\left|a \theta^{\prime}+b \psi^{\prime}\right|<$ $\left|\theta^{\prime}\right|$ hold; thus, $\theta$ and $\psi$ are adjacent minima.

Thus, if $\mathcal{S}=\langle 1, \psi\rangle$ where $\psi>0$ and $\psi^{\prime}<-1$, we see by the above theorem that $\mathcal{S}$ must be a reduced lattice and $\psi+\left[-\psi^{\prime}\right]$ must be the minimum adjacent to 1 in $\delta$. If we put $\delta_{1}=\delta$, we have $\theta_{g}^{(1)}=\psi+\left[-\psi^{\prime}\right]$; also, by Theorems 2.2 and 3.1, we have $\theta_{g}^{(n+1)}=1 / \theta_{g}^{(n)}+\left[-1 / \theta_{g}^{(n)}\right]$. Thus, if we put $\phi=\phi_{0}=-\psi^{\prime}, \quad q_{0}=\left[\phi_{0}\right], \phi_{k}=$ $-1 / \theta_{g}^{(k) \prime}, q_{k}=\left[\phi_{k}\right]$, we find that

$$
\phi_{0}=\left[q_{0}, q_{1}, q_{2}, \ldots, q_{n-1}, \phi_{n}\right]
$$


is the RCF expansion of $\phi_{0}$. The regular continued fraction expansion of $\phi$, where $\phi>1, \phi^{\prime}<0$, is therefore equivalent to the chain (2.1) of minima in the lattice $\left\langle 1,-\phi^{\prime}\right\rangle$. In fact $\theta_{n}=A_{n-2}-\phi^{\prime} B_{n-2}$.

We will now give a similar interpretation of the NICF algorithm in $\S$. We first let $\mathcal{S}=\langle 1, \mu\rangle$ be reduced and let $\chi \in \mathcal{S}$ be defined by

$$
\chi= \begin{cases}\theta & \text { when } \theta^{\prime}>-\frac{1}{2} \\ \omega & \text { when } \theta^{\prime}<-\frac{1}{2}\end{cases}
$$

where $\theta(>1)$ is the minimum adjacent to 1 in $\delta$ and $\omega(>\theta)$ is the minimum adjacent to $\theta$ in $\delta$.

Algorithm 1. Put $\mathcal{T}_{1}=\mathcal{S}_{1}$ and let $\chi_{g}^{(n)}$ be the value of $\chi$ in $\mathcal{T}_{n}$. Define $\mathcal{T}_{n+1}=$ $\left(1 / \chi_{g}^{(n)}\right) \mathcal{T}_{n}$.

We see that the lattices $\mathcal{T}_{i}$ are all reduced by Theorem 2.3; further, if

$$
\chi_{n}=\prod_{i=1}^{n-1} \chi_{g}^{(i)}
$$

then $\chi_{n} \mathcal{T}_{n}=\mathcal{T}_{1}$.

THEOREM 3.2. In (3.1) above, we have $\omega=\theta+1$.

Proof. Clearly $\mathcal{S}=\langle 1, \theta\rangle=\langle\theta, \theta+1\rangle$. Further, $0<\theta^{\prime}+1<\frac{1}{2}$; hence, $\left|\theta^{\prime}+1\right|$ $<\left|\theta^{\prime}\right|$ and $\left(\theta^{\prime}+1\right) \theta^{\prime}<0$. The theorem now follows from Theorem 3.1.

THEOREM 3.3. If $\mathcal{S}=\langle 1, \psi\rangle$, where $\left|\psi^{\prime}\right|\left\langle\frac{1}{2}, \psi\right\rangle 1$, and $\delta$ is a reduced lattice, then $\chi$ in (3.1) must be $\psi$.

Proof. If $\psi^{\prime}<0$, then $\theta$ in (3.1) must be $\psi$ by Theorem 3.1. Also, since $\psi^{\prime}>-\frac{1}{2}$, we have $\chi=\theta=\psi$. If $\psi^{\prime}>0$, then $-1<\psi^{\prime}-1<-\frac{1}{2}$ and $\delta=\langle 1, \psi-1\rangle$. Since $\mathcal{S}$ is reduced, we must have $\theta=\psi-1$, and $\theta^{\prime}<-\frac{1}{2}$. It follows that $\chi=\omega=\theta+1=\psi$ by Theorem 3.2 .

THEOREM 3.4. Let $\psi>1$ and $\left|\psi^{\prime}\right|<\frac{1}{2}$. If we put $\phi=\operatorname{sgn}\left(\psi^{\prime}\right) / \psi$ and $\chi=N e\left(\phi^{\prime}\right)-\phi$, we must have $\chi>1$ and $\left|\chi^{\prime}\right|<\frac{1}{2}$.

Proof. Clearly $\left|\chi^{\prime}\right|<\frac{1}{2}$. Also, since $\psi>1$, we have $|\phi|<1$. Now if $\psi^{\prime}<0$, then $-\frac{1}{2}<\psi^{\prime}<0$; hence, $1 / \psi^{\prime}<-2$ and $\phi^{\prime}>2$. We have $\chi>2-\phi>1$. Similarly, $\chi>1$ when $\psi^{\prime}>0$.

Suppose that $\mathcal{T}_{1}=\mathcal{S}_{1}=\langle 1, \psi\rangle$, where $\psi>0$ and $\psi^{\prime}<-1$. We have $\chi_{g}^{(1)}=\psi-$ $N e\left(\psi^{\prime}\right)$ by Theorem 3.3. Also, by Theorems 3.3 and 3.4, $\chi_{g}^{(i+1)}=N e\left(\phi_{i}^{\prime}\right)-\phi_{i}$, where $\phi_{i}=\eta_{i} / \chi_{g}^{(i)}$ and $\eta_{i}=\operatorname{sgn}\left(\chi_{g}^{(i) \prime}\right)$. If we put $\rho_{0}=-\psi^{\prime}$ and $\rho_{i}=\left(\prod_{j=1}^{i} \eta_{j}\right) \phi_{i}^{\prime}$, we have

$$
\rho_{i+1}=\left(\prod_{j=1}^{i} \eta_{j}\right) / \chi_{g}^{(i+1) \prime}=\left(\prod_{j=1}^{i} \eta_{j}\right) /\left(N e\left(\phi_{i}^{\prime}\right)-\phi_{i}\right)=1 /\left(N e\left(\rho_{i}\right)-\rho_{i}\right) .
$$

Thus, the problem of expanding $\phi$ into a NICF when $\phi>1$ and $\phi^{\prime}<0$ is equivalent to the problem of finding the values of $\chi_{n}$ in (3.2) when $\mathcal{T}_{1}=\mathcal{S}_{1}=\left\langle 1,-\phi_{1}^{\prime}\right\rangle$. Note that $\left|\rho_{i}^{\prime}\right|=1 / \psi_{g}^{(i)}$; also, $\chi_{n}=\left|\overline{A_{n-2}}-\phi^{\prime} \bar{B}_{n-2}\right|$.

We now have an interpretation of the NICF algorithm as a means of finding certain elements in the lattice $\mathcal{S}$. In the next section we will extend the NICF to the NIVCF by extending Algorithm 1 in $\delta$ to an algorithm analogous to it in $R$. 
4. The VCF and the NIVCF in $\Re$. Throughout this section and the remainder of this work we will assume that $\Re=\langle 1, \mu, \nu\rangle$ and that $R$ is a reduced lattice. We have seen that the problem of finding the elements of a chain (2.1) can be solved when we can solve the problem of finding the minimum adjacent to 1 in $\mathscr{L}$. When $\mathcal{L}=\mathcal{S}$ Theorem 3.1 provides us with an easy method for determining this minimum and this, in turn, leads to the RCF. When $\mathcal{L}=\Re$, however, the problem is somewhat more difficult. In [5] Voronoi showed how this could be done for the minimum adjacent to 1 and less than 1. In Section 33 of [5] he also gave a method for finding the minimum which is adjacent to 1 and greater than 1 , but did not provide a proof for his method. He stated that he had such a proof but that it was too complicated to give. In [8] a method, slightly different from that of Voronoi was given for doing this. In order to briefly describe this technique, we require some notation.

Let $\alpha \in \Re$. We define the puncture $P(\alpha)$ to be the point $\left(\xi_{\alpha}, \eta_{\alpha}\right)$ in the $x-y$ plane, where

$$
\xi_{\alpha}=\left(2 \alpha-\alpha^{\prime}-\alpha^{\prime \prime}\right) / 2, \quad \eta_{\alpha}=\left(\alpha^{\prime}-\alpha^{\prime \prime}\right) / 2 i .
$$

Further, we define $\zeta_{\alpha}=\left(\alpha^{\prime}+\alpha^{\prime \prime}\right) / 2$. We note that $\alpha=\zeta_{\alpha}+\xi_{\alpha}, \xi_{\alpha+\beta}=\xi_{\alpha}+\xi_{\beta}$, $\eta_{\alpha+\beta}=\eta_{\alpha}+\eta_{\beta}, \zeta_{\alpha+\beta}=\zeta_{\alpha}+\zeta_{\beta}$. Also, if $\alpha \in \mathcal{Q}$, we have $\eta_{\alpha}=\xi_{\alpha}=0$ and $\zeta_{\alpha}=\alpha$. Thus, the set of all punctures formed from all the points of $R$ also forms a lattice $\mathcal{L}^{(p)}$ with basis $\{P(\mu), P(\nu)\}$. We also point out that if $\xi_{\alpha}$ or $\eta_{\alpha}$ is rational, then $\delta$ must be the zero of both a quadratic and cubic polynomial with integer coefficients unless $\alpha \in 2$. Since $\alpha-[\alpha] \in \Re, 0 \leqslant \alpha-[\alpha]<1$, and $0 \leqslant \alpha^{\prime}-[\alpha]<1$ when $\alpha \in \mathcal{2}$, we see that, since $\Re$ is reduced, we must have $\alpha=[\alpha]$. Thus, if $\xi_{\alpha}$ or $\eta_{\alpha}$ is rational, then $\alpha \in \mathbf{Z}$ and $\xi_{\alpha}=\eta_{\alpha}=0$.

In [8] it is shown that there exist $\phi, \psi \in \Re$ such that

$$
\xi_{\phi}>\xi_{\psi}>0 ; \quad \eta_{\phi} \eta_{\psi}<0, \quad\left|\eta_{\psi}\right|>\left|\eta_{\phi}\right|
$$

and

$$
\left|\eta_{\psi}\right|>\sqrt{3} / 4, \quad\left|\eta_{\phi}\right|<1-\sqrt{3} / 4, \quad 2\left|\eta_{\psi}\right|>1-\left|\eta_{\phi}\right| .
$$

Further such $\phi$ and $\psi$ can be found by using the RCF in $\varrho^{(p)}$ as described in [2] or [8]. Actually, there exist $\phi, \psi$ in $\Re$ such that $\xi_{\phi}>\xi_{\psi}>0, \eta_{\phi} \eta_{\psi}<0,\left|\eta_{\phi}\right|<\frac{1}{2}$, $\left|\eta_{\psi}\right|>\frac{1}{2}$, but these may be difficult for a computer to find because of precision problems; hence, the more relaxed conditions given above are preferable for computational purposes. In [8] the following theorem is proved.

THEOREM 4.1. If $\theta(>1)$ is the minimum adjacent to 1 in $\Re$, then $P(\theta)$ must be one of $P(\phi), P(\psi), P(\phi-\psi), P(\phi+\psi)$ or $P(2 \phi+\psi)$.

Thus, once $\phi$ and $\psi$ have been found there are only a few choices for $P(\theta)$. We need only find that $\theta$ such that $P(\theta)$ is one of these five choices, $\left|\theta^{\prime}\right|<1$ and $\theta(>0)$ is least. Since

$$
\left|\alpha^{\prime}\right|^{2}=\eta_{\alpha}^{2}+\zeta_{\alpha}^{2}
$$

we must have $\left|\zeta_{\alpha}\right|<1$. Hence $\theta$ must be $a+\gamma$, where $\gamma$ is in $\{\phi, \psi, \phi-\psi, \phi+\psi$, $2 \phi+\psi\}$ and $a=\left[-\zeta_{\gamma}\right]$ or $\left[-\zeta_{\gamma}\right]+1$. It is then necessary to find the least of these 10 possibilities for $\theta$ such that $\left|\theta^{\prime}\right|<1$. By evaluating the integer $I=N(\theta-N(\theta))$ this latter condition can be checked by using only integer arithmetic. We have $\left|\theta^{\prime}\right|<1$ if and only if $I<0$. 
It is of some interest to know how frequently we might expect $P(\theta)$ to be $P(\phi)$, $P(\psi), P(\phi-\psi), P(\phi+\psi)$ or $P(2 \phi+\psi)$. To this end we computed all the lattices $\Re_{i}(i=1,2,3, \ldots, p)$ where $\Re_{1}=\langle 1, \mu, \nu\rangle$ and $\{1, \mu, \nu\}$ is a basis of $\Theta_{\mathscr{F}}$ for each distinct pure cubic field $\mathscr{F}=2(\sqrt[3]{D})$ with $D \leqslant 67,000$. We determined a total of 233762826 lattices and found that $P(\theta)=P(\phi) 137074347$ times, $P(\theta)=P(\psi)$ 70776182 times, $P(\theta)=P(\phi-\psi) 24432636$ times, $P(\theta)=P(\phi+\psi) 1479661$ times, and $P(\theta) \neq P(2 \phi+\psi)$. One might be led to conclude from this large amount of evidence that $P(\theta)$ is never $P(2 \phi+\psi)$, but when $D=68781$ we find that in $\Omega_{2307}$, $\phi=\left(-72036+1809 \delta+2 \delta^{2}\right) / 126539$, and $\psi=\left(117574-2668 \delta+67 \delta^{2}\right) / 126539$. We have $\theta=\left(-26498+950 \delta+71 \delta^{2}\right) / 126539$ and $P(\theta)=P(2 \phi+\psi)$. It certainly appears that this case is a rarity and, as such, seems to be a nuisance to consider. In fact, as we shall see from results in this section and Lemma 7.2, we can find $\varepsilon_{0}$ by ignoring this case. We may not find all the $\Re_{i}$ and $\theta_{i}$ this way but we will find $\varepsilon_{0}$.

In order to develop the NIVCF, we use the idea of Algorithm 1 in Section 3 and extend it to $R$. The value of $\theta^{\prime}$ for $\theta$ in $\delta$ represents the last coordinate of the corresponding point $\Theta$ of the lattice $\mathcal{S}$. The analogous quantity for $\theta \in \mathcal{R}$ is $\zeta_{\theta}=\left(\theta^{\prime}+\theta^{\prime \prime}\right) / 2$. If $\theta(>1)$ is the minimum of $\mathcal{R}$ adjacent to 1 and $\omega(>\theta)$ is the minimum of $R$ adjacent to $\theta$, we define

$$
\chi= \begin{cases}\theta & \text { when } \theta^{\prime}+\theta^{\prime \prime}>-1 \\ \omega & \text { when } \theta^{\prime}+\theta^{\prime \prime}<-1\end{cases}
$$

This is analogous to the definition (3.1) of $\chi$ in $\S$. We also point out here that $\theta^{\prime}+\theta^{\prime \prime}<-1$ if and only if $N\left(\theta^{\prime}+\theta^{\prime \prime}+1\right)<0$. Now if $T=\theta+\theta^{\prime}+\theta^{\prime \prime}, S=\theta \theta^{\prime}$ $+\theta^{\prime} \theta^{\prime \prime}+\theta^{\prime \prime} \theta, N=\theta \theta^{\prime} \theta^{\prime \prime}$, then $T, S, N \in \mathbf{Z}$ and $N\left(\theta^{\prime}+\theta^{\prime \prime}+1\right)<0$ if and only if $N(T+1-\theta)<0$. Since $N(T+1-\theta)=T^{2}+2 T+1+(T+1) S-N$, we can use integer arithmetic only to test whether or not $\theta^{\prime}+\theta^{\prime \prime}<-1$.

We now have

The NIVCF Algorithm. Put $\mathcal{T}_{1}=\mathscr{R}_{1}$, and let $\chi_{g}^{(n)}$ be the value of $\chi$ in $\sigma_{n}$. Define $\widetilde{T}_{n+1}=\left(1 / \chi_{g}^{(n)}\right) \mathcal{T}_{n}$.

We have

$$
\chi_{n}=\prod_{i=1}^{n-1} \chi_{g}^{(i)}
$$

and

$$
x_{n} \widetilde{T}_{n}=\widetilde{T}_{1} .
$$

This, of course, is just the same as Algorithm 1 except that $\mathscr{T}_{1}=\mathscr{R}_{1}$ and we use (4.2) to define $\chi$.

When $\theta^{\prime}+\theta^{\prime \prime}<-1$, we have $\left|\theta^{\prime}+1\right|<\left|\theta^{\prime}\right|$ and $\theta+1>\theta$; thus, $\chi=\omega \leqslant \theta+1$. Unfortunately, we cannot, as we did in Section 3, prove that $\omega=\theta+1$; for this is not always the case. In the next few sections we will develop an algorithm for finding $\omega$ when $\theta^{\prime}+\theta^{\prime \prime}<-1$. In the remainder of this section we will develop some results concerning our NIVCF algorithm. These results are analogous to some of those presented in Section 1. We first require some simple lemmas.

LEMMA 4.2. If $\chi_{s}=\theta_{n}$, then $\mathcal{T}_{s}=\mathcal{R}_{n}$. 
Proof. From (2.3), we have $\theta_{n} \Re_{n}=\Re_{1}$, and from (4.4) we have $\chi_{s} \mathcal{T}_{s}=\mathcal{T}_{1}$; thus, $\theta_{n} \Re_{n}=\chi_{s} \mathcal{T}_{s}$ and since $\chi_{s}=\theta_{n}$, we must have $\mathcal{T}_{s}=\mathcal{R}_{n}$.

LEMMA 4.3. If $\chi_{s}=\theta_{n}$, then $\chi_{s+1}=\theta_{n+1}$ or $\theta_{n+2}$.

Proof. From Lemma 4.2 we have $\mathcal{T}_{s}=\Re_{n}$; thus, $\theta$ in $\mathcal{T}_{s}$ is $\theta_{g}^{(n)}$. If $\theta_{g}^{(n) \prime}+\theta_{g}^{(n) \prime \prime}>$ -1 , then $\chi_{g}^{(s)}=\theta_{g}^{(n)}$; if $\theta_{g}^{(n) \prime}+\theta_{g}^{(n) \prime \prime}<-1$, then $\chi_{g}^{(s)}=\omega=\theta_{g}^{(n)} \theta_{g}^{(n+1)}$ by Theorem 2.3. The lemma now follows easily from (2.2) and (4.3).

COROLlaRY. Given any $\chi_{s}$, there exists some $\theta_{n}(n \geqslant s)$ such that $\chi_{s}=\theta_{n}$.

Proof. Since $\chi_{1}=\theta_{1}=1$, the result follows by using the lemma and induction on $s$.

COROllaRY. Given any pair $\theta_{n}$ and $\theta_{n+1}$ from (2.1), at least one of them must be some $\chi_{s}$ with $s \leqslant n+1$.

Proof. By induction on $n$.

Notice that these results are analogous to results, which are proved in [7], concerning the NICF.

LEMMA 4.4. Let $\rho \in \mathcal{Z}(\delta), 0<\rho<2 m$ and $\left|\rho^{\prime}\right|<m$, then $|d(\rho)|<c_{2} m^{6}$, where $d(\rho)=\left(\rho-\rho^{\prime}\right)^{2}\left(\rho^{\prime}-\rho^{\prime \prime}\right)^{2}\left(\rho^{\prime \prime}-\rho\right)^{2}$ and $c_{2}=9(1047+85 \sqrt{153}) / 128 \simeq 147.543$.

Proof. Put $c=\rho, a+b i=\rho^{\prime}, a-b i=\rho^{\prime \prime}$, where $a, b, c$ are real. Then $\left|\rho^{\prime}\right|^{2}=a^{2}$ $+b^{2}<m^{2},|a|<m,|b|<m$, and

$$
\sqrt{|d(\rho)|}=2|b|\left((a-c)^{2}+b^{2}\right) .
$$

Since $|d(\rho)| \leqslant 4 b^{2}\left(c^{2}+b^{2}\right)^{2}<100 m^{6}<c_{2} m^{6}$ when $a \geqslant 0$, we need only consider the case of $a<0$. We note that since $b^{2}<m^{2}-a^{2}$ and $|c-a|<2 m-a$, we have

$$
(a-c)^{2}+b^{2}<(2 m-a)^{2}+m^{2}-a^{2}=5 m^{2}-4 a m
$$

for values of $x$ such that $|x|<m$, the function $f(x)=4\left(m^{2}-x^{2}\right)\left(5 m^{2}-4 x m\right)^{2}$ has a single maximum at $x=(5-\sqrt{153}) \mathrm{m} / 16$; thus, $f(x)<c_{2} m^{6}$ when $|x|<m$. It follows that $|d(\rho)|<c_{2} m^{6}$ when $-m<a<0$.

We now prove a result which is analogous to Theorem 1.3. Part of the proof of this result is based on an idea which Arne Brentjes (personal communication) used to extend Theorem 1 of [9]. We assume that $\{1, \mu, \nu\}$ is a basis of $\vartheta_{\mathscr{F}}$ and $R=\langle 1, \mu, \nu\rangle$.

THEOREM 4.5. Let $\alpha=x+y \mu+z \nu$, where $\operatorname{gcd}(x, y, z)=1$, if $\alpha>1$ and $N(\alpha)$ $<\sqrt[4]{|\Delta| / c_{2}}$, then $\alpha=\chi_{k}$ for some $k>1$.

Proof. Certainly $\alpha \neq \chi_{1}=1$. Assume $\alpha \neq \chi_{k}$ for any $k>1$. Since $\alpha>1$, there must be some integer $n \geqslant 1$ such that

$$
\theta_{n} \leqslant \alpha<\theta_{n+1}
$$

in the chain (2.1) when $\theta_{1}=1$. If $\alpha \neq \theta_{n}$, put $\rho=N(\alpha) \theta_{n} / \alpha$. We have $\rho \in \theta_{\mathscr{f}}$, $0<\rho<N(\alpha)$ and $\left|\rho^{\prime}\right|<N(\alpha)$, the latter result following from the fact that $\alpha$ is not the minimum adjacent to $\theta_{n}$. If $\alpha=\theta_{n}$, then, since $\alpha \neq \chi_{k}$ for any $k \geqslant 1$, we must have $\theta_{n+1}=\chi_{k}$ for some $k \geqslant 1$ by a corollary of Lemma 4.3. In this case put $\rho=N(\alpha) \theta_{n+1} / \alpha=N(\alpha) \theta_{g}^{(n)}$. Now $\left|\theta_{g}^{(n) \prime}\right|<1$ and $\chi_{g}^{(k-1)}=\theta_{g}^{(n-1)} \theta_{g}^{(n)} \leqslant \theta_{g}^{(n-1)}+1$; thus, $\left|\rho^{\prime}\right|<N(\alpha)$ and $0<\rho \leqslant N(\alpha)\left(1+1 / \theta_{g}^{(n-1)}\right)<2 N(\alpha)$. It follows from the 
above observations that if $\alpha \neq \chi_{k}$ for any $k>1$, there must exist some $\rho \in \theta_{\mathscr{\sigma f}}$ such that $0<\rho<2 N(\alpha),\left|\rho^{\prime}\right|<N(\alpha)$. Further, since $\rho=N(\alpha) \beta / \alpha$, where $\alpha \neq \beta$ and $\beta=\theta_{n}$ or $\theta_{n+1}$, we cannot have $d(\rho)=0$. For, if $d(\rho)=0$, then $\rho \in \mathbf{Z}$ as $\rho \in \theta_{\mathscr{F}}$. Since $\beta=a+b \mu+c \nu$, where $a, b, c \in \mathbf{Z}$, we get $x=N(\alpha) a / \rho . y=N(\alpha) b / \rho$, $z=N(\alpha) c / \rho$. Since $\operatorname{gcd}(x, y, z)=1$ and $\operatorname{gcd}(a, b, c)=1$ (otherwise $\beta$ could not be a minimum of $\mathcal{R})$, we get $\rho=N(\alpha)$ and $\beta=\alpha$, which is not so.

Now

$$
\begin{aligned}
d(\rho) & =N(\alpha)^{6}\left(\frac{\beta}{\alpha}-\frac{\beta^{\prime}}{\alpha^{\prime}}\right)^{2}\left(\frac{\beta^{\prime}}{\alpha^{\prime}}-\frac{\beta^{\prime \prime}}{\alpha^{\prime \prime}}\right)^{2}\left(\frac{\beta^{\prime \prime}}{\alpha^{\prime \prime}}-\frac{\beta}{\alpha}\right)^{2} \\
& =\frac{N(\alpha)^{6}}{\left(\alpha \alpha^{\prime} \alpha^{\prime \prime}\right)^{4}}\left|\begin{array}{ccc}
\alpha^{2} & \alpha \beta & \beta^{2} \\
\alpha^{\prime 2} & \alpha^{\prime} \beta^{\prime} & \beta^{\prime 2} \\
\alpha^{\prime \prime 2} & \alpha^{\prime \prime} \beta^{\prime \prime} & \beta^{\prime \prime 2}
\end{array}\right|^{2}
\end{aligned}
$$

Since $\alpha, \beta \in \vartheta_{\mathscr{F}}$, we have $A=\left(a_{i j}\right)_{3 \times 3}$, where $a_{i j} \in \mathbf{Z}$ such that

$$
\left(\alpha^{2}, \alpha \beta, \beta^{2}\right)=(1, \mu, \nu) A \text {. }
$$

Thus, we have

$$
\left|\begin{array}{ccc}
\alpha^{2} & \alpha \beta & \beta^{2} \\
\alpha^{\prime 2} & \alpha^{\prime} \beta^{\prime} & \beta^{\prime 2} \\
\alpha^{\prime \prime 2} & \alpha^{\prime \prime} \beta^{\prime \prime} & \beta^{\prime \prime 2}
\end{array}\right|=\left|\begin{array}{lll}
1 & \mu & \nu \\
1 & \mu^{\prime} & \nu^{\prime} \\
1 & \mu^{\prime \prime} & \nu^{\prime \prime}
\end{array}\right||A|
$$

and $|d(\rho)|=N(\alpha)^{2}|A|^{2}|\Delta|$.

Since $d(\rho) \neq 0$, we have $|A| \neq 0$ and $|A| \in \mathbf{Z}$. By Lemma 4.4, $|d(\rho)|<c_{2} N(\alpha)^{6}$; hence, $c_{2} N(\alpha)^{4}>|A|^{2}|\Delta|$. We see that

$$
N(\alpha)>\sqrt[4]{|A|^{2}|\Delta| / c_{2}} \geqslant \sqrt[4]{|\Delta| / c_{2}}
$$

and this is impossible. Thus $\alpha=\chi_{k}$ for some $k \geqslant 1$.

We also have a corollary which is analogous to Theorem 1.2.

COROLlaRY. If $\Delta<-23$, then $\varepsilon_{0}+\chi_{k}$ for some $k>1$; if $\Delta=-23$, then $\varepsilon_{0}^{2}=\chi_{2}$.

Proof. $N\left(\varepsilon_{0}\right)=1$ and $1<\sqrt[4]{|\Delta| / c_{2}}$ for $|\Delta|>c_{2}$. Thus we need only test directly the few cases (see, for example, [2]) where $|\Delta|<c_{2}$. For each of these cases we find that $\varepsilon_{0}=\chi_{k}$ for some $k>1$ except for the case of $\Delta=-23$ where $\chi_{2}=\varepsilon_{0}^{2}$.

Note that if $\pi=k-1$ in this corollary, we have $\mathcal{T}_{\pi+1}=\mathcal{T}_{1}$ and the NIVCF algorithm is periodic with period length $\pi$. We also note that the NIVCF algorithm can be used to find all of the solutions $\alpha \in \vartheta_{\mathscr{F}}$ of the Diophantine equation

$$
N(\alpha)=I
$$

when $|I|<\sqrt[4]{|\Delta| / c_{2}}$.

5. Geometric Inequalities. In order to find $\chi$ in any reduced lattice $R$ we need to be able to find $\omega$ when $\theta^{\prime}+\theta^{\prime \prime}<-1$. We will do this by limiting the number of possibilities for $P(\omega)$. In order to do this we require the inequalities from plane Euclidean geometry which are presented in this section. For our further work the most important of these results are Lemmas 5.4, 5.5, 5.6 and 5.7. However, in order 
to prove these results we require three simple lemmas which we give here without proof. We say that $P$ is in a certain geometric figure if it is either on the boundary of that figure or within it.

Lemma 5.1. If $P$ is any point in the triangle $A B C$, then $\overline{A P} \leqslant \max (\overline{A B}, \overline{A C})$.

The next two lemmas refer to Figure 1 below.

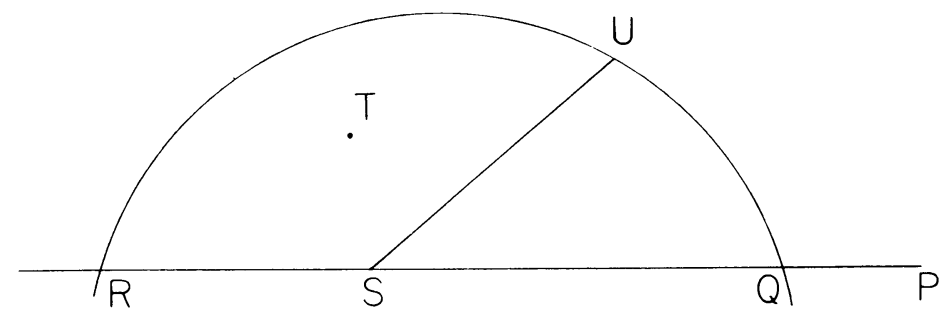

Figure 1

Here $R Q$ is a chord of a circle and $U$ lies on the smaller of the arcs cut off by $R Q$ or on either of them if $R Q$ is a diameter of the circle.

LEMMA 5.2. If $T$ is any point in the segment $R U Q$ in Figure 1 and $P$ is any point on the chord $R Q$ produced, then $\overline{P T} \leqslant \overline{P R}$.

LEMMA 5.3. If $T$ is any point in the region bounded by the lines $R S, S U$, and the arc $R U$, then $\overline{S T} \leqslant \max (\overline{R S}, \overline{S U})$.

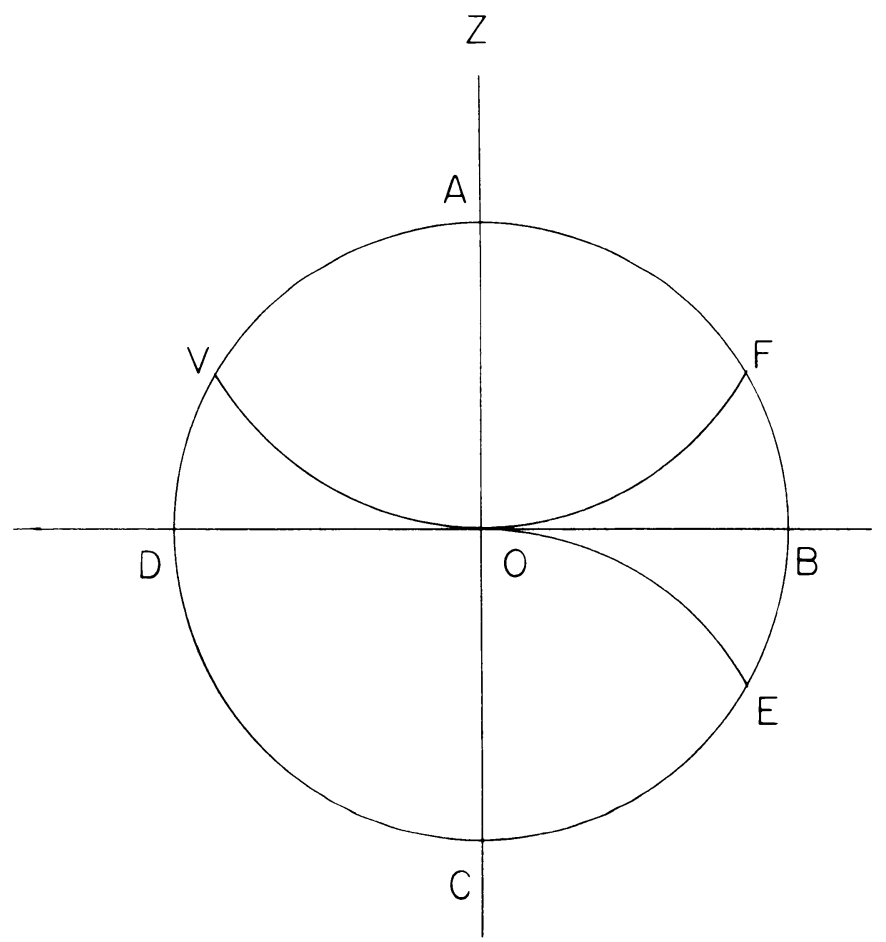

FIGURE 2 
In the lemmas that follow we will refer to a figure (Figure 2) which we now describe. Let $O Z(z)$ and $O Y(y)$ be perpendicular axes. The circle centre $O$ and radius $\overline{O A}=1$ cuts the positive $z$ axis at $A$, and the positive $y$ axis at $B$, the negative $z$ and $y$ axes at $C$ and $D$, respectively. The arc $V O F$ is described by a circle with centre $A$ and radius $1 . F$ is the point where this arc meets the $\operatorname{arc} A B$ and $V$ is the point where this arc meets the arc $A D$. Also, the arc $O E$ is described by a circle with centre $C$ and radius 1 and $E$ is the point where this arc meets the arc $B C$. We denote that figure bounded by the line $O C$ and the arcs $O F$ and $F C$ by the symbol $\Theta$.

We now consider the figure $A B C$ (see Figure 3 ) which is bounded by three circular arcs with centres $A, B, C$, the vertices of an equilateral triangle, and radii equal to $\overline{A B}$.

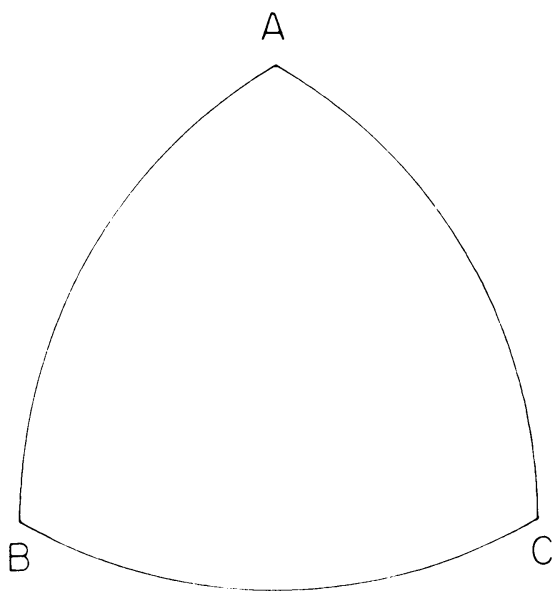

Figure 3

This is the Reuleaux triangle and is well known as a figure of constant breadth. As such it follows that if $P, Q$ are any two points in the Reuleaux triangle above, we must have $\overline{P Q} \leqslant \overline{A B}$. Since the figure $Q$ is completely covered by two Reuleaux triangles with vertices at $O, F, E$ and $O, C, E$, we have the following lemma.

LEMMA 5.4. If $P, Q$ are any two points in $Q$ and $\overline{P Q}>1$, then one of the points must lie above the arc $O E$ and the other must lie below the arc $O E$. Further, if $R$ is any other point of $Q$, then $\min (\overline{P R}, \overline{Q R}) \leqslant 1$.

Lemma 5.5. Let $P, Q$ be any two points in the semicircle $A B C$ of Figure 2 such that $\overline{P Q}=1$ and $P Q$ is parallel to $A C$. If $R$ is any point in this same semicircle such that $\overline{O R} \leqslant \max (\overline{O P}, \overline{O Q})$, then $\min (\overline{P R}, \overline{Q R}) \leqslant 1$.

Proof. We refer to Figure 4 below.

We may assume with no loss of generality that $\overline{O P} \geqslant \overline{O Q}$. If $P=(a, b), Q=(a$, $b-1)$, then since both $P, Q$ are in the semicircle, we have $\frac{1}{2} \leqslant b \leqslant 1$ and $0 \leqslant a$ $\leqslant \sqrt{3} / 2$. If $R=(r, s)$ and $|s| \geqslant \frac{1}{2}$, then $R, Q$ are in the Reuleaux triangle $O C E$ or $R$, $P$ are in the Reuleaux triangle $O F A$. In either case we get $\min (\overline{P R}, \overline{Q R}) \leqslant 1$. We now assume $|s|<\frac{1}{2}$. Let $\bigodot_{1}$ and $\bigodot_{2}$ be circles with radii 1 and centres $P$ and $Q$, 


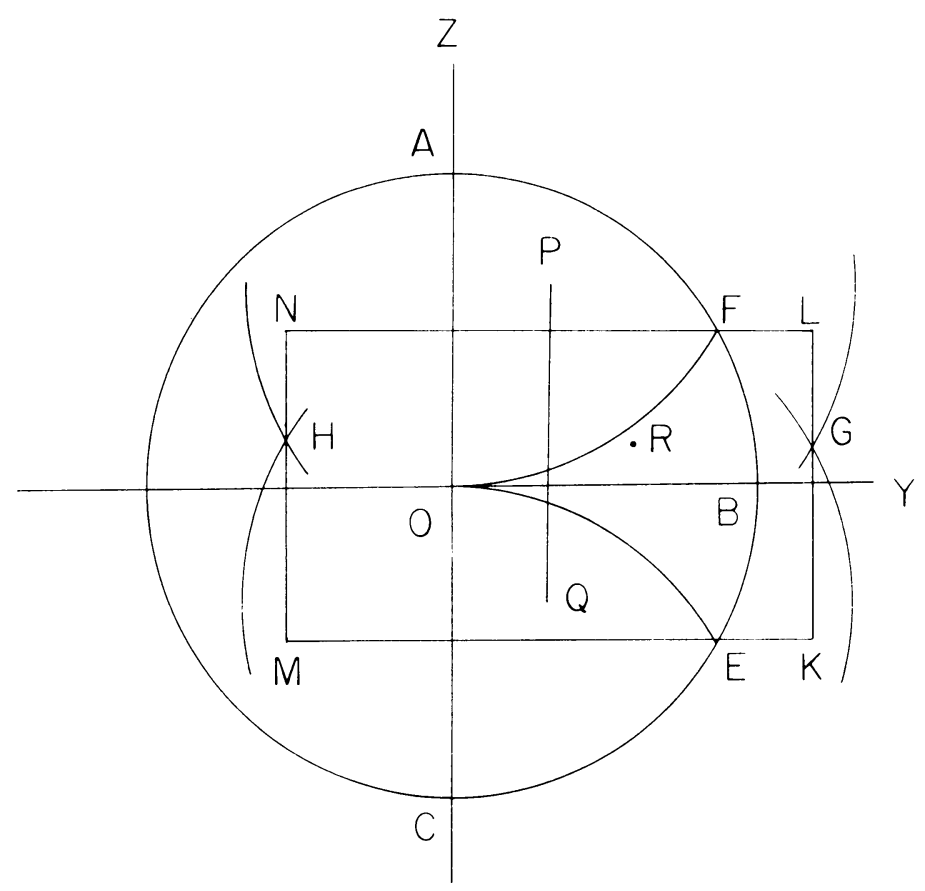

FIGURE 4

respectively. $C_{1}$ and $C_{2}$ intersect at points $G=\left(a+\sqrt{3} / 2, b-\frac{1}{2}\right)$ and $H=(a$ $\left.-\sqrt{3} / 2, b-\frac{1}{2}\right)$. Let $K=\left(a+\sqrt{3} / 2,-\frac{1}{2}\right), L=\left(a+\sqrt{3} / 2, \frac{1}{2}\right), M=(a-\sqrt{3} / 2$, $\left.-\frac{1}{2}\right), N=\left(a-\sqrt{3} / 2, \frac{1}{2}\right)$. The rectangle $L K M N$ is covered by the circles $\bigodot_{1}$ and $e_{2}$; thus, if $R$ lies in this rectangle, the lemma is certainly true. In order, then, to prove the lemma completely, we need only show that it holds when $r>a+\sqrt{3} / 2$. If $r>a+\sqrt{3} / 2$ and $s \geqslant b-\frac{1}{2}$, then $r^{2}+s^{2} \leqslant a^{2}+b^{2}, b s \geqslant b^{2}-b / 2$, and $r a>a^{2}$ $+a \sqrt{3} / 2 ;$ also,

$$
\begin{aligned}
\overline{P R}^{2} & =(a-r)^{2}+(b-s)^{2}=a^{2}+b^{2}+r^{2}+s^{2}-2(a r+b s) \\
& <2 a^{2}+2 b^{2}-2 b^{2}+b-2 a^{2}-\sqrt{3} a \\
& =b-\sqrt{3} a \leqslant 1 .
\end{aligned}
$$

If $r>a+\sqrt{3} / 2$ and $s<b-\frac{1}{2}$, we have $1-b \geqslant 0,-s>\frac{1}{2}-b$, and $s(b-1)>$ $(1-b)\left(\frac{1}{2}-b\right)$. Thus

$$
\overline{P Q}^{2}=(a-r)^{2}+(b-1-s)^{2}<b-\sqrt{3} a \leqslant 1 .
$$

Thus, one of $\overline{P Q}$ or $\overline{P R}$ must be less than 1 and the lemma is proved.

LEMMA 5.6. In Figure 2 let $L$ be a point on $O B$ such that $\overline{O L}=\lambda=\sqrt{(5-\sqrt{5}) / 8}$. Let $K L M$ be a line perpendicular to $O B$, meeting the arc $A B$ at $K$, the arc $O F$ at $G$, the arc $O E$ at $H$, and the arc $B C$ at $M$. Let $P$ be any point on the figure bounded by the line $G H$ and the arcs $O G$ and $O H$. If $Q$ is in the Figure $Q$ and $\overline{O P} \geqslant \overline{O Q}$, then $\overline{P Q} \leqslant 1$.

Proof. We refer to Figure 5 below. 


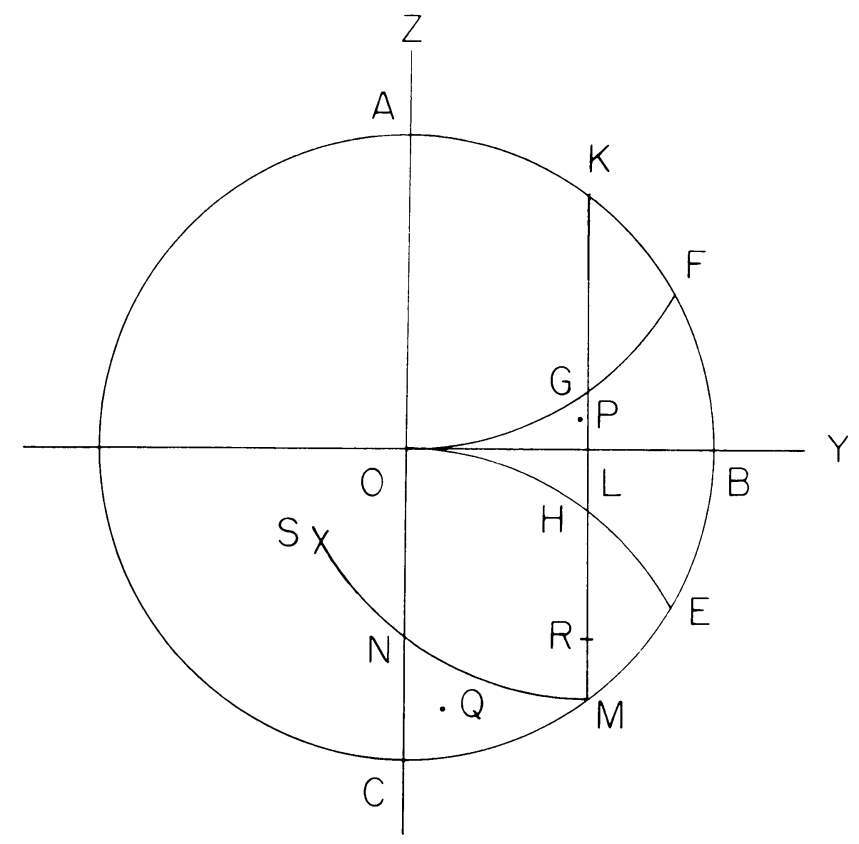

Figure 5

We first note that $\overline{G M}=\overline{O C}=1$. Let $S$ be that point such that $\overline{S M}=\overline{S G}=\overline{G M}$ and $S$ is to the left of the $z$-axis. Let the arc $S M$ with radius 1 and centre $G$ cut $O C$ at $N$. If $Q$ lies in the Reuleaux triangle $M S G$, we have $\overline{P Q} \leqslant 1$. If $Q$ lies above the arc $O E$, we have $\overline{P Q} \leqslant 1$. If $Q$ lies below the arc $O E$ and to the right of the line $K L M$, then $\overline{O Q}>\overline{O H}=\overline{O G} \geqslant \overline{O P}$, which is not so. Thus, we need only consider $Q$ as lying on the figure bounded by the line $N C$ and the arcs $N M$ and $C M$. Put $g=\overline{G L}, a=\overline{O G}, b=\overline{O N}$. We have $\lambda^{2}+g^{2}=a^{2}$ and $(1-g)^{2}+\lambda^{2}=1$. It follows that $2 g=a^{2}$ and $g=1 \pm \sqrt{1-\lambda^{2}}=1 \pm(1+\sqrt{5}) / 4$. Since $g<\frac{1}{2}$, we get $g=$ $(3-\sqrt{5}) / 4$ and $a=(\sqrt{5}-1) / 2$. If $\overline{R M}=\overline{G L}$, then $\overline{L R}=\overline{O N}, 2 g+b=1$, and we find that $b=a$. Since we need not consider $Q$ to be $N$, we have $\overline{O Q}>\overline{O N}=\overline{O G}$ $\geqslant \overline{O P}$, which is not possible

LEMMA 5.7. Let $Q$ be any point in $Q$ and let $R$ be the point lying below $Q$ such that $Q R \| A C$ and $\overline{Q R}=1$. Let $P$ be any point of $Q$ such that $P$ lies below the arc $O E$ and $\overline{P Q}>1, \overline{P R}>1$. Let $S$ be that point such that $\overline{S A}=\overline{P Q}, \overline{S O}=\overline{P R}$ and $S$ is on the other side of $A C$ from $P$. If $T$ is any point in that region of the semicircle $A D C$ below or on the arc $V O$, then $\min (\overline{P T}, \overline{S T}) \leqslant 1$.

\section{Proof. Refer to Figure 6 below.}

We first note that since $\overline{P Q}>1, Q$ must, by Lemma 5.4, lie above the arc $O E$. Let $U$ be the point below $S$ such that $\frac{U S}{P S}=1, U S \| A C$ and let $M$ be the point above $P$ such that $\overline{P M}=1$ and $P M \| A C$. We see that $S M P U, M P R Q$, and $A S U O$ are parallelograms. Also, $M$ lies in the Reuleaux triangle $A O F$, and, since $\overline{O U}=\overline{S A}$ $=\overline{P Q}>1, U$ lies outside the circle $A B C D$. Let $M P$ and $Q R$ meet $O B$ at $N$ and $W$, respectively. Since $\overline{M Q}=\overline{P R}>1$, if follows from Lemma 5.5 that $\overline{O Q} \geqslant \overline{O P}$. If 


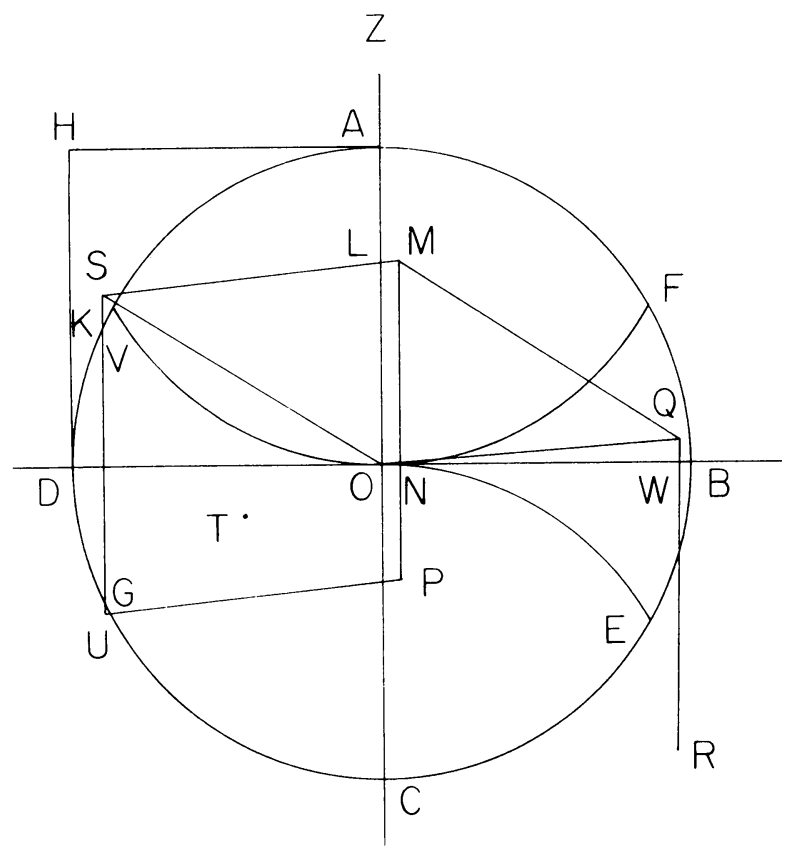

FIGURE 6

$\overline{O N} \geqslant \overline{O W}$, then $\overline{N P} \leqslant \overline{Q W}$ and this means that $P$ and $Q$ must lie in the same Reuleaux triangle $O F E$, an impossibility. Hence $\overline{O N}<\overline{O W}$ and $O S M Q$ is a parallelogram with $M S \| O Q$ and $\overline{M S}=\overline{O Q} \leqslant 1$.

Since $M$ lies in the Reuleaux triangle $A O F$, the angles $O A M$ and $A O M$ cannot be obtuse. Let $S M$ meet $A C$ at $L$. We have $\overline{S L} \leqslant \overline{S M} \leqslant 1$ and $\overline{S O}>1, \overline{S A}>1$; hence, the angles $S A M$ and $S O M$ must be acute. If $L$ lies above $A$ on the $z$-axis, then, since the angles $L A M$ and $O A M$ are complementary, we see that the angle $S A M$ cannot be acute, a contradiction. Similarly, $L$ cannot lie below $O$ on the $z$-axis. Since $L$ must lie between $O$ and $A$ on $O A$, we see that the angles $S A L$ and $S O L$ are acute. Since the perpendicular distance of $S$ to $P M$ is the same as that from $Q$ to $A O$, we also see that $S$ must lie in the square $D O A H$. Indeed, since $\overline{O S}=\overline{P R}>1, S$ must lie in the triangle $D H A$.

Suppose $T$ lies in that segment of the circle $A B C D$ which is cut off by the chord formed by producing $U P$. Suppose further that $T$ lies under this chord. Since $U$ and $P$ both lie below or on $D B$ and on opposite sides of $A C$, by Lemma 5.3 we get $\overline{P T} \leqslant \max (\overline{P C}, \overline{U P})$. Since $\overline{U P}=\overline{S M}=\overline{O Q} \leqslant 1$ and $P$ and $C$ are in the Reuleaux triangle $O C E$, we get $\overline{P T} \leqslant 1$.

If $T$ lies in the parallelogram $S U P M$, then by Lemma 5.1, either $\overline{P T}$ or $\overline{S T}$ must be less than or equal to $\max (\overline{U P}, \overline{P M})=1$. If $T$ lies in the segment $K D G$ which is cut off the circle $A B C D$ by $S U$, then, by Lemma $5.2 \overline{S T} \leqslant \overline{S G}<\overline{S U}=1$.

Since $S$ must lie in the triangle $D H A$, we must have $\overline{H S} \leqslant 1$ by Lemma 5.1. Thus, if $T$ lies in the triangle $H S L$, then $\overline{S T} \leqslant 1$ by Lemma 5.1. If $T$ lies in the triangle $H A L$, then $T$ lies in the triangle $H A O$ and this means that $T$ must lie above the arc $V O$, which is not so. Since there are no other possible regions of the semicircle $A D C$ in which $T$ could be, the lemma is proved. 
6. Some Lemmas Concerning the Reduced Lattice $(R$. In order to complete our development of the NIVCF algorithm in Section 8 we will make use of a number of lemmas concerning any reduced lattice $R=\langle 1, \mu, \nu\rangle$. We note that if $\theta \in \mathscr{R}$, $\left|\theta^{\prime}\right| \leqslant 1,\left|\theta^{\prime}-1\right|>1$ and $\eta_{\theta}>0$, then $P=\left(\eta_{\theta}, \zeta_{\theta}\right)$ is a point in c' of Section 5 .

Lemma 6.1. If $\omega, \chi \in$ ?R; $\left|\omega^{\prime}\right|,\left|\chi^{\prime}\right| \leqslant 1$; and $2 \chi=\omega+1$, then $\left|\chi^{\prime}-1\right| \leqslant 1$.

Proof. We have $2 \zeta_{\chi}=\zeta_{\omega}+1$ and $\eta_{\omega}=2 \eta_{\chi}$. Further, from (4.1), $\zeta_{\omega}^{2}+\eta_{\omega}^{2}=\left|\omega^{\prime}\right|^{2}$ $\leqslant 1$ and $\left|\zeta_{\omega}\right| \leqslant 1$. It follows that

$$
1+\zeta_{\omega}^{2}+\eta_{\omega}^{2}+2\left|\zeta_{\omega}\right| \leqslant 4
$$

and, as a consequence, $\left(\zeta_{\chi}-1\right)^{2}+\eta_{\chi}^{2} \leqslant 1$. Hence $\left|\chi^{\prime}-1\right| \leqslant 1$.

Lemma 6.2. Let $\omega, \chi, \psi \in \mathfrak{G}$, where $\omega, \chi, \psi$ are all distinct $\left|\omega^{\prime}\right|,\left|\chi^{\prime}\right|,\left|\psi^{\prime}\right| \leqslant 1$, and $\left|\omega^{\prime}-1\right|,\left|\chi^{\prime}-1\right|,\left|\psi^{\prime}-1\right|>1$. If $\eta_{\omega} \eta_{\chi}>0$ and $\eta_{\chi} \eta_{\psi}>0$, we cannot have any $b$ such that $b<\omega, \chi, \psi<b+1$.

Proof. By symmetry we may assume with no loss of generality that $P=\left(\eta_{\omega}, \zeta_{\omega}\right)$, $Q=\left(\eta_{\chi}, \zeta_{\chi}\right), R=\left(\eta_{\psi}, \zeta_{\psi}\right)$ are all points in ( $\psi$. By Lemma 5.4, two of these points, say $Q$ and $R$, are such that $\overline{Q R} \leqslant 1$. Thus, $\left|\chi^{\prime}-\psi^{\prime}\right| \leqslant 1$. If $b<\chi, \psi<b+1$, then $|\chi-\psi|<1$, and we can only have $\chi=\psi$, a contradiction.

Lemma 6.3. Let $\theta, \omega \in$ ?h such that $\left|\theta^{\prime}\right| \leqslant 1,\left|\omega^{\prime}\right| \leqslant 1, \omega>\theta>1$, and $\left|\omega^{\prime}\right|<\left|\theta^{\prime}\right|$. If $\eta_{\omega} \eta_{\theta}>0$ and $\left|\theta^{\prime}+1\right| \leqslant 1$, then $\omega \geqslant \theta+1$.

Proof. Suppose $\omega<\theta+1$. Since $\omega>\theta$, we have $0<\omega-\theta<1$ and $-1<\omega-\theta$ $-1<0$. Since $\eta_{\omega} \eta_{\theta}>0$ and $\left|\theta^{\prime}+1\right| \leqslant 1$, we may assume with no loss of generality that $P=\left(\eta_{\theta}, \zeta_{\theta}+1\right), Q=\left(\eta_{\theta}, \zeta_{\theta}\right), R=\left(\eta_{\omega}, \zeta_{\omega}\right)$ in Figure 4. Since $\left|\omega^{\prime}\right|=\overline{O R}<\overline{O P}$ $=\left|\theta^{\prime}\right|$, we must have one of $\overline{Q R}=\left|\omega^{\prime}-\theta^{\prime}\right|$ or $\overline{P R}=\left|\omega^{\prime}-\theta^{\prime}-1\right|$ less than or equal to 1 by Lemma 5.5. Since $\omega-\theta, \omega-\theta-1 \in \Re$, we can only have $\omega=\theta$ or $\omega=\theta+1$. The latter is impossible and the former is also impossible since $\left|\omega^{\prime}\right|<\left|\theta^{\prime}\right|$. Thus, we must have $\omega \geqslant \theta+1$.

Lemma 6.4. Let $\theta, \omega \in$ oR such that $\omega>\theta>1 ;\left|\omega^{\prime}\right|,\left|\theta^{\prime}\right| \leqslant 1 ;\left|\theta^{\prime}-1\right|,\left|\omega^{\prime}-1\right|>1$; and $\eta_{\omega} \eta_{\theta}>0$. If $\left|\theta^{\prime}\right|>\left|\omega^{\prime}\right|$ and $\left|\eta_{\theta}\right|<\lambda$, then $\omega \geqslant \theta+1$.

Proof. If $\left|\theta^{\prime}+1\right| \leqslant 1$, then by Lemma 6.3 , we have $\omega \geqslant \theta+1$. Thus, we may assume $\left|\theta^{\prime}+1\right|>1$. With no loss of generality, we may also assume $P=\left(\eta_{\theta}, \zeta_{\theta}\right)$, $Q=\left(\eta_{\omega}, \zeta_{\omega}\right)$ in Figure 5. Since $\overline{O P}=\left|\theta^{\prime}\right|>\left|\omega^{\prime}\right|=\overline{O Q}$, we must, by Lemma 5.6, have $\overline{P Q}=\left|\theta^{\prime}-\omega^{\prime}\right| \leqslant 1$. If $\theta<\omega<\theta+1$, we find that $\theta-\omega=0$, which is not possible; thus, $\omega \geqslant \theta+1$.

Lemma 6.5. Let $\theta, \omega, \chi \in \Re$ such that $\left|\theta^{\prime}\right| \leqslant 1,\left|\omega^{\prime}\right| \leqslant 1,\left|\omega^{\prime}+1\right| \leqslant 1,\left|\omega^{\prime}-1\right|>1$, $\left|\theta^{\prime}-1\right|>1,\left|\chi^{\prime}-1\right|>1, \eta_{\theta} \eta_{\omega}<0, \eta_{\omega} \eta_{\chi}>0$. If $\left|\omega^{\prime}-\chi^{\prime}\right|>1$ and $\left|\omega^{\prime}-\chi^{\prime}+1\right|>1$, then either $\left|\theta^{\prime}-\omega^{\prime}+\chi^{\prime}-1\right| \leqslant 1$ or $\left|\theta^{\prime}-\omega^{\prime}\right| \leqslant 1$.

Proof. With no loss of generality we may assume that $T=\left(\eta_{\theta}, \zeta_{\theta}\right), P=\left(\eta_{\omega}, \zeta_{\omega}\right)$, $Q=\left(\eta_{\chi}, \zeta_{\chi}\right), R=\left(\eta_{\chi}, \zeta_{\chi}-1\right)$ in Figure 6. Since $\overline{P Q}=\left|\omega^{\prime}-\chi^{\prime}\right|>1$ and $\overline{P K}=\mid \omega^{\prime}$ $-\chi^{\prime}+1 \mid>1$ and $S=\left(\eta_{\omega}-\eta_{\chi}, \zeta_{\omega}-\zeta_{\chi}+1\right)$, we must have $\overline{S T}=\mid \theta^{\prime}-\omega^{\prime}+\chi^{\prime}-$ $1 \mid$ or $\overline{P T}=\left|\omega^{\prime}-\theta^{\prime}\right|$ less than or equal to 1 by Lemma 5.7 . 
Lemma 6.6. Let $\theta, \omega, \chi \in G R$ and let $\theta(>1)$ be the minimum adjacent to 1 . Suppose that $\left|\omega^{\prime}\right| \leqslant 1,\left|\chi^{\prime}\right| \leqslant 1,\left|\theta^{\prime}+1\right| \leqslant 1,\left|\omega^{\prime}+1\right| \leqslant 1, \eta_{\omega} \eta_{\theta}<0, \eta_{\omega} \eta_{\chi}>0$. If $\theta<\chi, \omega<\theta+1$, then $\theta=\omega+1-\chi$.

Proof. Since $0<\chi-1, \omega-1<\theta$, we see by the reasoning of Lemma 2.4 that $\left|\chi^{\prime}-1\right|,\left|\omega^{\prime}-1\right|>1$.

If $\left|\omega^{\prime}-\chi^{\prime}\right|,\left|\omega^{\prime}-\chi^{\prime}+1\right|>1$, then, by Lemma 6.5 , either $\left|\omega^{\prime}-\theta^{\prime}\right| \leqslant 1$ or $\mid \theta^{\prime}-\omega^{\prime}$ $+\chi^{\prime}-1 \mid \leqslant 1$. If $\left|\omega^{\prime}-\theta^{\prime}\right| \leqslant 1$, then since $|\omega-\theta|<1$, we get $\omega=\theta$ which is not so. If $\left|\theta^{\prime}-\omega^{\prime}+\chi^{\prime}-1\right| \leqslant 1$, we see that $\left|\rho^{\prime}\right| \leqslant 1$, where $\rho=\theta-\omega+\chi-1$. Now $\theta+\chi>\theta+1>\omega$ and $\chi-(\omega+1)<0$; thus, $-1<\rho<\theta$. It follows that $\rho$ can only be 0 or 1 . But if $\rho=0$, then $\theta=\omega-\chi+1$ and this is impossible because $\left|\omega^{\prime}-\chi^{\prime}+1\right|>1$. If $\rho=1$, then $\theta=\omega-\chi+2$. Since $\left|\omega^{\prime}-\chi^{\prime}+1\right|>1$ and $-3 / 2$ $<\zeta_{\omega}-\zeta_{\chi}<1 / 2$, we cannot have $\left|\theta^{\prime}\right|<1$; thus, $\rho \neq 1$.

If $\left|\omega^{\prime}-\chi^{\prime}\right| \leqslant 1$, then $\omega=\chi$, which is not so. Hence, we must assume that $\left|\omega^{\prime}-\chi^{\prime}+1\right| \leqslant 1$ and $\left|\omega^{\prime}-\chi^{\prime}\right|>1$. Put $\rho=\omega-\chi+1$. We have $\left|\rho^{\prime}\right|<1$ and $0<\rho<1+1<1+\theta$. Since we cannot have $\rho<\theta$, we have $\theta \leqslant \rho<1+\theta$. If $\rho=\theta$, we have the lemma; thus, we will assume that $\theta<\rho<1+\theta$. By the reasoning of Lemma 2.4 , we have $\left|\rho^{\prime}-1\right|>1$.

Suppose $\eta_{\rho} \eta_{\omega}>0$. If $\rho=\omega$, we get $\chi=1$, an impossibility. If $\rho=\chi$, then $2 \chi=\omega+1$. Since $\left|\chi^{\prime}-1\right|>1$, this cannot be true by Lemma 6.1. Thus, $\rho, \chi, \omega$ are all distinct elements of $\mathscr{R}$ such that $\eta_{\rho}, \eta_{\omega}, \eta_{\chi}$ have the same sign; $\left|\rho^{\prime}\right|,\left|\omega^{\prime}\right|,\left|\chi^{\prime}\right| \leqslant 1$; $\left|\rho^{\prime}-1\right|,\left|\omega^{\prime}-1\right|,\left|\chi^{\prime}-1\right|>1 ; \theta<\rho, \omega, \chi<\theta+1$. By Lemma 6.2, this is impossible.

If $\eta_{\rho}=0$, then $\omega-\chi \in \mathbf{Z}$. Since $|\omega-\chi|<1$, this means that $\omega=\chi$, which is not so. We can only have $\eta_{\rho} \eta_{\omega}<0$ and, as a consequence, $\eta_{\rho} \eta_{\theta}>0$. Since $0<\rho-\theta<1$ and $-1<\rho-\theta-1<0$, we see that $\left|\theta^{\prime}-\rho^{\prime}\right|>1$ and $\left|\theta^{\prime}-\rho^{\prime}+1\right|>1$. Since $\left|\theta^{\prime}+1\right| \leqslant 1$, we get $\left|\chi^{\prime}-\theta^{\prime}\right| \leqslant 1$ or $\left|\chi^{\prime}-\theta^{\prime}+\rho^{\prime}-1\right| \leqslant 1$ by Lemma 6.5. Since $0<\chi-\theta<1$, we cannot have $\left|\chi^{\prime}-\theta^{\prime}\right| \leqslant 1$. Since $\chi-\theta+\rho-1=\omega-\theta$, we also cannot have $\left|\chi^{\prime}-\theta^{\prime}+\rho^{\prime}-1\right| \leqslant 1$. It follows that $\rho=\theta$.

If $\omega \in \mathscr{R}, \omega \notin \mathcal{Q}$ and $P(\omega)=\left(\xi_{\omega}, \eta_{\omega}\right)$ is the puncture of $\omega$, let $\omega^{*}$ be defined as that element of $R$ such that $P\left(\omega^{*}\right)=P(\omega),\left|\omega^{* \prime}\right| \leqslant 1$ and $\left|\omega^{*}\right|$ is minimal. We note here that if $\left|\eta_{\omega}\right|>1$, then $\omega^{*}$ cannot exist. Since there must exist $a \in \mathbf{Z}$ such that $\left|\zeta_{\omega}-a\right| \leqslant \frac{1}{2}$, we see that if $\left|\eta_{\omega}\right|<\sqrt{3} / 2$, then $\eta_{\omega}^{2}-\left(\zeta_{\omega}-a\right)^{2}<1$. That is, if $\left|\eta_{\omega}\right|$ $<\sqrt{3} / 2$, then $\omega^{*}$ exists. We also point out that if $\omega^{*}$ exists and $\omega^{*}>0$, then $\left|\omega^{* \prime}-1\right|>1$. For if $\left|\omega^{* \prime}-1\right| \leqslant 1$, then $\omega^{*}>1$ and $\left|\omega^{*}-1\right|<\left|\omega^{*}\right|$. Since $P\left(\omega^{*}\right)=$ $P\left(\omega^{*}-1\right)$, this contradicts the definition of $\omega^{*}$.

We require the following lemma of [8] (Lemma 4.3).

LEMma 6.7. Let $P(\chi)=\left(\xi_{\chi}, \eta_{\chi}\right)$ be the puncture of $\chi \in \Re$ such that $\xi_{\chi}>0,\left|\eta_{\chi}\right|$ $<\sqrt{3} / 2$, and let $P(\omega)=\left(\xi_{\omega}, \eta_{\omega}\right)$.be the puncture of $\omega \in \Re$. If $\left|\omega^{\prime}\right| \leqslant 1, \xi_{\omega}>\xi_{\chi}$, and $\eta_{\omega} \eta_{\chi}>0$, then $\omega>\chi^{*}$.

LEMMA 6.8. Let $\theta(>1)$ be the minimum of $R$ adjacent to 1 and let $\omega(>\theta)$ be the minimum of $R$ adjacent to $\theta$. Suppose that $\left|\theta^{\prime}+1\right| \leqslant 1$ and $\omega<\theta+1$. If there exists $\chi \in \Re$ such that $\left|\eta_{\chi}\right|<\sqrt{3} / 2, \xi_{\omega}>\xi_{\chi}>0, \eta_{\omega} \eta_{\chi}>0$, then $\chi^{*}=\omega+1-\theta$.

Proof. Since $\left|\eta_{\chi}\right|<\sqrt{3} / 2, \chi^{*}$ exists. Further, since $\left|\chi^{*}\right|>1$ and $\chi^{*}=\xi_{\chi}+\zeta_{\chi^{*}}$ with $\xi_{\chi}>0$ and $\zeta_{\chi^{*}} \geqslant-1$, we have $\chi^{*}>1$. Since $\eta_{\chi} \eta_{\omega}>0, \xi_{\omega}>\xi_{\chi}$, and $\left|\omega^{\prime}\right| \leqslant 1$, 
we must have $\omega>\chi^{*}$ by Lemma 6.7 . Since $\omega$ is a minimum of $R$, it follows that $\left|\chi^{* \prime}\right|>\left|\omega^{\prime}\right|$. Since $\left|\chi^{*^{\prime}}\right|<1$ and $\chi^{*}>0$, we must have $\chi^{*}>\theta$. If $\chi^{*} \geqslant \theta+1$, then $\omega>\theta+1$, which is not so; thus, $1<\theta<\chi^{*}<\omega<\theta+1<\chi^{*}+1$. Further, $\left|\chi^{* \prime}-1\right|>1$ and $\left|\omega^{\prime}-1\right|>1$.

If $\left|\chi^{*}+1\right| \leqslant 1$, by Lemma 6.3 , we get $\omega>\chi^{*}+1$, which is impossible. Since $\left|\omega-\chi^{*}\right|<1$, we must have $\left|\omega^{\prime}-\chi^{* \prime}\right| \geqslant 1$. By Lemma 5.4, we must have $\left|\omega^{\prime}+1\right| \leqslant 1$. If $\eta_{\theta} \eta_{\omega}>0$, then $\omega \geqslant \theta+1$ by Lemma 6.3. Thus, $\eta_{\theta} \eta_{\omega}<0$ and $\theta=\omega+1-\chi^{*}$ by Lemma 6.6.

7. The Main Result. In this section we will show how to limit the possible values for $\omega$ in $R$. We assume that we have found $\phi, \psi \in \Re$ and a basis $\{P(\phi), P(\psi)\}$ of $\mathcal{L}^{(p)}$ with the properties given in Section 4 . We require simple results concerning elements of $\Re$ which have certain punctures in $\mathcal{L}^{(p)}$. Throughout this section and the next we denote by $\theta(>1)$ the minimum adjacent to 1 in $R$ and by $\omega(>\theta)$ the minimum adjacent to $\theta$ in $R$.

LEMMA 7.1. If $\zeta_{\theta}<-\frac{1}{2}, \omega \neq \theta+1, \theta \neq \phi^{*}, \omega \neq \phi^{*}$, and either $\xi_{\theta}>\xi_{\phi}$ or $\eta_{\theta} \eta_{\phi}<0$, then $\xi_{\phi}>\xi_{\omega}$.

Proof. Since $\left|\eta_{\phi}\right|<\sqrt{3} / 2, \phi^{*}$ exists. Further, since $\xi_{\phi}>0$, we have $\phi^{*}>1$. Since $\left|\phi^{* \prime}\right| \leqslant 1$, we must have $\phi^{*}>\theta$. If $\xi_{\theta}>\xi_{\phi}$ and $\eta_{\theta} \eta_{\phi}>0$, then $\theta>\phi^{*}$ by Lemma 6.7; thus, we may assume $\eta_{\theta} \eta_{\phi}<0$. Since $\eta_{\omega} \eta_{\theta}<0$ (see the proof of Lemma 6.8), we also have $\eta_{\omega} \eta_{\phi}>0$. If $\xi_{\omega}>\xi_{\phi}$, we have $\omega>\phi^{*}$ by Lemma 6.7 ; thus, since $\omega$ is a minimum, $\left|\omega^{\prime}\right|<\left|\phi^{* \prime}\right|$. If $\omega>\phi^{*}$, then, since $\left|\eta_{\phi}\right|<\lambda$, we must have $\omega \geqslant \phi^{*}+1>\theta$ +1 by Lemma 6.4. Since $\zeta_{\theta}<-\frac{1}{2}$, this is impossible.

LEMMA 7.2. If $P(\theta)=2 P(\phi)+P(\psi)$, then $\zeta_{\theta}<-\frac{1}{2}$.

Proof. Since $\left|\eta_{\phi}\right|<1-\sqrt{3} / 4<\sqrt{7} / 4$, we have

$$
\sqrt{1-\eta_{\phi}^{2}}>3 / 2-\sqrt{1-\eta_{\phi}^{2}}
$$

Since $\left|\phi^{* \prime}-1\right|>1$, we have $\zeta_{\phi^{*}}<1-\sqrt{1-\eta_{\phi}^{2}}$. Since $\phi^{*}>1$ and $\phi^{*}=\xi_{\phi}+\zeta_{\phi^{*}}$, we see that $\xi_{\phi}>\frac{1}{2}+\zeta_{\phi^{*}}$. Since $\theta<\phi^{*}$, we have $\zeta_{\theta}+2 \xi_{\phi}+\xi_{\psi}<\zeta_{\phi^{*}}+\xi_{\phi}$; hence, $\zeta_{\theta}<\zeta_{\theta}+\xi_{\psi}<\zeta_{\phi^{*}}-\xi_{\phi}<-\frac{1}{2}$.

LEMMA 7.3. If $P(\theta)=2 P(\phi)+P(\psi)$, then $\theta<\phi^{*}<\theta+1,\left|\theta^{\prime}\right|>\left|\phi^{* \prime}\right|$, and $\zeta_{\phi^{*}}>$ 0 .

Proof. Since $\theta=\zeta_{\theta}+\xi_{\theta}<\phi^{*}=\zeta_{\phi^{*}}+\xi_{\phi}$, we have $\zeta_{\phi^{*}}>\xi_{\phi}+\zeta_{\theta}$. Also, $\zeta_{\phi^{*}}+\xi_{\phi}$ $=\phi^{*}>1$; thus, $\zeta_{\phi^{*}}>1-\zeta_{\phi^{*}}+\zeta_{\theta}$ and $2 \zeta_{\phi^{*}}>1+\zeta_{\theta}>0$. Since $\left|\eta_{\phi}\right|<\sqrt{3} / 2$ and $\left|\phi^{* \prime}-1\right|>1$, we must have $0<\zeta_{\phi^{*}}<\frac{1}{2}$; further, $\xi_{\phi}>1-\zeta_{\phi^{*}}>\frac{1}{2}>\zeta_{\phi^{*}}$. Thus, $\zeta_{\theta}<\zeta_{\phi^{*}}-\xi_{\phi}<0$ and $\left|\zeta_{\theta}\right|>\xi_{\phi}-\zeta_{\phi^{*}}>1-2 \zeta_{\phi^{*}}>2 \sqrt{1-\eta_{\phi}^{2}}-1$.

Now $\theta<\phi^{*}<2 \xi_{\phi}<1+\zeta_{\theta}+2 \xi_{\phi}+\xi_{\psi}=1+\theta$. Since $-\frac{1}{2}<-\zeta_{\phi^{*}}<1-\zeta_{\phi^{*}}$ $+\zeta_{\theta}<\zeta_{\phi^{*}}<\frac{1}{2}$, we must have $\left|\eta_{\theta}-\eta_{\phi}\right|>\sqrt{3} / 2$. Hence, $\left|\eta_{\psi}\right|>\sqrt{3} / 2+\left|\eta_{\phi}\right|>2\left|\eta_{\phi}\right|$ 
and $\eta_{\theta} \eta_{\phi}<0$. Thus, $\left|\eta_{\theta}\right|>\sqrt{3} / 2-\left|\eta_{\phi}\right|$ and

$$
\begin{aligned}
\left|\theta^{\prime}\right|^{2} & =\eta_{\theta}^{2}+\zeta_{\theta}^{2}>\left(\sqrt{3} / 2-\left|\eta_{\phi}\right|\right)^{2}+\left(2 \sqrt{1-\eta_{\phi}^{2}}-1\right)^{2} \\
& >(\sqrt{3} / 2-(1-\sqrt{3} / 4))^{2}+\left(2 \sqrt{1-(1-\sqrt{3} / 4)^{2}}-1\right)^{2} \\
& >(1-\sqrt{3} / 4)^{2}+\left(1-\sqrt{1-(1-\sqrt{3} / 4)^{2}}\right)^{2} \\
& >\eta_{\phi}^{2}+\zeta_{\phi^{*}}^{2} \\
& =\left|\phi^{* \prime}\right|^{2} .
\end{aligned}
$$

LEMMA 7.4. If $P(\theta)=P(\phi)+P(\psi)$ and $\zeta_{\theta}<-\frac{1}{2}$, then $P(\omega) \neq P(\phi)-P(\psi)$.

Proof. Assume that $P(\omega)=P(\phi)-P(\psi)$. If $\phi^{*}>\theta+1$, then $\zeta_{\phi^{*}}+\xi_{\phi}>\zeta_{\theta}+\xi_{\phi}$ $+\xi_{\psi}+1$ and $\zeta_{\phi^{*}}>\zeta_{\theta}+1+\xi_{\psi}>0$. If $\psi^{*}$ exists, then $\xi_{\psi}>1-\zeta_{\psi^{*}}>\frac{1}{2}$ and $\zeta_{\phi^{*}}$ $>\frac{1}{2}$, which is not possible. If $\psi^{*}$ does not exist, we must have $\left|\eta_{\psi}\right|>\sqrt{3} / 2$. Consider $\chi=\phi^{*}-\omega$. Since $P(\chi)=P(\psi)$, we see that $\left|\phi^{* \prime}-\omega^{\prime}\right|>1$; for, othersie, $\psi^{*}$ would exist. Also $\left|\eta_{\omega}\right|=\left|\eta_{\phi}-\eta_{\psi}\right|=\left|\eta_{\phi}\right|+\left|\eta_{\psi}\right|>\left|\eta_{\phi}\right|+\sqrt{3} / 2$ and $\zeta_{\phi^{*}}>0$. Thus $P=\left(\eta_{\phi}\right.$, $\left.\zeta_{\phi^{*}}\right)$ and $Q=\left(\eta_{\omega}, \zeta_{\omega}\right)$ must lie in the same Reuleaux triangle FOE in Figure 2 and $\left|\phi^{* \prime}-\omega^{\prime}\right|=\overline{P Q} \leqslant 1$, a contradiction.

Thus, we must have $\phi^{*} \leqslant \theta+1$. Since $P\left(\phi^{*}\right) \neq P(\theta)$, we can only have $\phi^{*}<\theta+$ 1 ; hence, $\theta<\phi^{*}, \omega<\theta+1, \eta_{\theta} \eta_{\omega}<0$ and $\eta_{\phi} \eta_{\omega}>0$. One of $\left|\omega^{\prime}+1\right|$ or $\left|\phi^{* \prime}+1\right|$ must be less than or equal to 1 ; for if this were not the case we would see that the points $P, Q$ above would be such that $\left|\phi^{* \prime}-\omega^{\prime}\right|=\overline{P Q} \leqslant 1$, which is not possible. Thus, by Lemma 6.6 , we get $\theta=\omega+1-\phi^{*}$ or $\theta=\phi^{*}+1-\omega$. Since $P(\theta) \neq$ $-P(\psi)$ and $P(\theta) \neq P(\psi)$, neither of these is possible; hence, $P(\omega) \neq P(\phi)-P(\psi)$.

In the theorem below we are able to give the possibilities for the puncture of $\omega$ when $\zeta_{\theta}<-\frac{1}{2}$.

THEOREM 7.5. When $\zeta_{\theta}<-\frac{1}{2}$, the puncture of $\omega, P(\omega)$, must be one of the punctures given in the second column of the table below.

TABLE 7.1

\begin{tabular}{|l|l|}
\hline$P(\theta)$ & possible values of $P(\omega)$ \\
\hline \hline$P(\phi)$ & $P(\phi), P(\psi), P(\phi+\psi)$ \\
\hline$P(\psi)$ & $P(\phi), P(\psi), P(\phi-\psi)$ \\
\hline$P(\phi-\psi)$ & $P(\psi), P(\phi-\psi)$ \\
\hline$P(\phi+\psi)$ & $P(\phi), P(\phi+\psi)$ \\
\hline$P(2 \phi+\psi)$ & $P(\phi)$ \\
\hline
\end{tabular}

Proof. By Theorem 4.1 we know that $P(\theta)$ must be one of the 5 possible entries in the first column of Table 7.1. Since $P(\omega) \in \mathcal{L}^{(p)}$, we must have

$$
P(\omega)=a P(\phi)+b P(\psi)
$$


where $a, b \in \mathbf{Z}$. That is,

$$
\begin{aligned}
& \xi_{\omega}=a \xi_{\phi}+b \xi_{\psi}, \\
& \eta_{\omega}=a \eta_{\phi}+b \eta_{\psi} .
\end{aligned}
$$

Further, we note that since $\theta, \omega$ are minima of $ᄋ$, we have $\left|\omega^{\prime}-1\right|>1,\left|\theta^{\prime}-1\right|>1$; also, $\left|\theta^{\prime}+1\right| \leqslant 1$ and $\xi_{\omega}>1-\zeta_{\omega}>0, \omega \leqslant \theta+1,\left|\omega^{\prime}\right|<\left|\theta^{\prime}\right|$. When $\omega \neq \theta+1$, we have $\eta_{\omega} \eta_{\theta}<0$ by Lemma 6.3. Suppose $a<0$ in (7.1). Since $\xi_{\omega}>0$, we must have $b>0$ and $\left|\eta_{\omega}\right|=|a|\left|\eta_{\phi}\right|+b\left|\eta_{\psi}\right|$ from (7.3). Since $\left|\eta_{\omega}\right|<1$, we have $b<2$. Since $\xi_{\phi}>\xi_{\psi}$, we must also have $b>|a|$. Thus, $b=1$ and $a=0$, which is not possible. Thus $a \geqslant 0$ in (7.1).

Case 1. $(P(\theta)=P(\phi))$. If $a=0$ in (7.1), then $\xi_{\omega}=b \xi_{\psi}$ and $\eta_{\omega}=b \eta_{\psi}$; thus, $0<b<3$. If $b=2$, then $\eta_{\omega} \eta_{\psi}>0, \xi_{\omega}>\zeta_{\psi}$ and $\left|\eta_{\psi}\right|<\frac{1}{2}<\sqrt{3} / 2$. It follows that $\psi^{*}$ exists and by Lemma $6.8 \psi^{*}=\omega+1-\theta$. Since $P(\psi)=P\left(\psi^{*}\right)=P(\omega-\theta)$, we get $P(\psi)=P(\theta)$, which is impossible for $a=0, b=2$. Thus, if $a=0$ we can only have $b=1$.

Suppose $a>0$. If $b \leqslant 0$, then from (7.3) we get $\eta_{\omega} \eta_{\phi}>0$ and $\eta_{\omega} \eta_{\theta}>0$. By Lemma 6.3, we can only have $\omega=\theta+1$ and $a=1, b=0$. If $b \geqslant a$, put $c=b-a$ $\geqslant 0$; we have, $\eta_{\omega}=a\left(\eta_{\phi}+\eta_{\psi}\right)+c \eta_{\psi}$ and $\left(\eta_{\phi}+\eta_{\psi}\right) \eta_{\psi}>0$. Thus, if $\left|\eta_{\phi}+\eta_{\psi}\right|>\frac{1}{2}$, then $\left|\eta_{\psi}\right|>\frac{1}{2}$. If either $a \geqslant 2$ or $c \geqslant 1$, we have $\left|\eta_{\omega}\right|>1$ which is not so. Thus $a=b=1$. If $\left|\eta_{\phi}+\eta_{\psi}\right|<\frac{1}{2}$, then there exists $\chi=(\phi+\psi)^{*}$. Since $\eta_{\omega} \eta_{\chi}>0$ and $\xi_{\omega}>\xi_{\chi}>0$ when $(a, b) \neq(1,1)$, we have (Lemma 6.8) $\chi=\omega+1-\theta$. Since this means that $P(\omega)=P(2 \phi+\psi)=2 P(\phi)+P(\psi)$, we get a contradiction. When $1 \leqslant b<a$, we have $\omega=\zeta_{\omega}+\xi_{\omega}=\zeta_{\omega}+a \xi_{\theta}+b \xi_{\psi}>2 \xi_{\theta}+\zeta_{\omega}>\xi_{\theta}+1-\zeta_{\theta}-1$ $>\xi_{\theta}+\zeta_{\theta}+1$. Thus, $\omega>\theta+1$ and this is impossible. We have proved the first row of Table 7.1. Before dealing with the remaining 4 cases we point out that if $P(\omega) \neq P(\phi), P(\theta) \neq P(\omega)$ and either $\eta_{\theta} \eta_{\phi}<0$ or $\xi_{\theta}>\xi_{\phi}$, then $\xi_{\phi}>\xi_{\omega}$ by Lemma 7.1.

Case 2. $(P(\theta)=P(\psi))$. If $a=0$, then $b>0$ and $\eta_{\theta} \eta_{\omega}=b \eta_{\psi}^{2}>0$; thus, by Lemma 6.3, we get $\omega \geqslant \theta+1$, which can only be so if $\omega=\theta+1$ and $(a, b)=(0,1)$. If $a>0$, we have $P(\theta) \neq P(\omega)$ and $\eta_{\theta} \eta_{\phi}=\eta_{\psi} \eta_{\phi}<0$. Thus, $\xi_{\phi}>\xi_{\omega}$ when $P(\omega) \neq$ $P(\phi)$. If $b>0$, then $P(\omega) \neq P(\phi)$ and $\xi_{\omega}>\xi_{\phi}$, which is not so. If $b=0$, then $\xi_{\omega}>\xi_{\phi}$ unless $a=1, b=0$. If $b<0$, we must have $|b| \leqslant 1$; otherwise, $\left|\eta_{\omega}\right|>1$. If $b=-1$ and $a>1$, then $P(\omega) \neq P(\phi)$ and $\xi_{\omega}>\xi_{\phi}$, thus $(a, b)=(1,-1)$.

Case 3. $(P(\theta)=P(\phi-\psi))$. Since $\zeta_{\theta}<-\frac{1}{2}$ and $\left|\theta^{\prime}\right|<1$, we must have $\left|\eta_{\theta}\right|$ $<\sqrt{3} / 2$. Thus, $\left|\eta_{\theta}\right|=\left|\eta_{\phi}\right|+\left|\eta_{\psi}\right|<\sqrt{3} / 2$ and $\left|\eta_{\psi}\right|<\sqrt{3} / 2$. If $P(\omega) \neq P(\theta)$, then $\eta_{\omega} \eta_{\theta}<0$. Further, $\eta_{\theta} \eta_{\phi}>0$ and $\eta_{\phi} \eta_{\psi}<0$; thus, $\eta_{\omega} \eta_{\psi}>0$ and $\eta_{\omega} \eta_{\phi}<0$. If $\xi_{\omega}>\xi_{\psi}$, we must have $\psi^{*}=\omega+1-\theta$ by Lemma 6.8. This means that $P(\omega)=P(\phi)$, which cannot be so as $\eta_{\omega} \eta_{\phi}<0$. Thus, $0<\xi_{\omega} \leqslant \xi_{\psi}$ and $0<a \xi_{\phi}+b \xi_{\psi} \leqslant \xi_{\psi}$ and $b<1$ or $(a, b)=(0,1)$. If $b=0$, then $\eta_{\omega} \eta_{\phi}>0$; hence, $b<0$. Since $1>\left|\eta_{\omega}\right|=a\left|\eta_{\phi}\right|+$ $|b|\left|\eta_{\psi}\right|$, we must have $b>-2$. Since $b=-1$ and $0<a \xi_{\phi}-\xi_{\psi}<\xi_{\psi}$, we see that $a$ can only be 1 and this contradicts $P(\omega) \neq P(\theta)$.

Case 4. $(P(\theta)=P(\phi+\psi))$. Assume $P(\omega) \neq P(\phi)$ and $P(\omega) \neq P(\theta)$. Since $\xi_{\theta}>$ $\xi_{\phi}$, we have $\xi_{\phi}>\xi_{\omega}$. If $a>0$, then, since $0<a \xi_{\phi}+b \xi_{\psi}<\xi_{\phi}$, we must have $b<0$. By the reasoning used in Case 2 we have $b=-1$ and $a=1$. By Lemma 7.4, this is not possible. Thus, we can only have $a=0$ and $b>0$. If this is so, we notice that $\eta_{\theta}=\eta_{\phi}+\eta_{\psi}$ and $\eta_{\omega}=b \eta_{\psi}$ have the same sign. By Lemma 6.3, this means that 
$\omega \geqslant \theta+1$. If $\omega=\theta+1$, we get a contradiction; thus, we can only have $P(\omega)=P(\phi)$ or $P(\omega)=P(\theta)=P(\phi+\psi)$.

Case 5. $(P(\theta)=P(2 \phi+\psi))$. By Lemma 7.3, we have $0<\phi^{*}<\theta+1$ and $\left|\phi^{* \prime}\right|<\left|\theta^{\prime}\right|$. If $\omega \neq \phi^{*}$, then $P(\omega) \neq P(\phi)$ and $\omega \neq \theta+1$. Thus, $\theta<\omega, \phi^{*}<\theta+1$. If $\eta_{\theta} \eta_{\phi}>0$, then, since $\xi_{\theta}>\xi_{\phi}$, we get $\theta>\phi^{*}$ by Lemma 6.7. Since this is not possible, we must have $\eta_{\theta} \eta_{\phi}<0$. Now $\eta_{\omega} \eta_{\theta}<0$; hence, $\eta_{\phi} \eta_{\omega}>0$. Using the reasoning of Lemma 7.4, we see that one of $\left|\omega^{\prime}+1\right|$ or $\left|\phi^{* \prime}+1\right|$ is less than or equal to 1 ; thus, by Lemma 6.6 , we have either $\theta=\phi^{*}+1-\omega$ or $\theta=\omega+1-\phi^{*}$. In the first case we put $P(\omega)=P(-\phi-\psi)$, an impossibility. In the second case we get $P(\omega)=P(3 \phi+\psi)$; but then $\xi_{\omega}>\xi_{\phi}$ and this cannot be so as $P(\omega) \neq P(\phi)$. Thus we can only have $P(\omega)=P\left(\phi^{*}\right)=P(\phi)$.

8. Implementation of the NIVCF Algorithm. We now describe how to find the possible values of $P(\omega)$ and then how $\omega$ itself can be found. We let $v$ be defined to be $\theta$ when $P(\theta) \neq P(2 \phi+\psi)$. When $P(\theta)=P(2 \phi+\psi)$ we define $v$ to be $\phi^{*}$. By Theorem 7.5, $\phi^{*}=\omega$ when $P(\theta)=P(2 \phi+\psi)$; thus, $v$ is that point of $R$ such that $P(v)$ is one of $P(\phi), P(\psi), P(\phi-\psi)$ or $P(\phi+\psi),\left|v^{\prime}\right| \leqslant 1, v>0$ and $v$ is the least point of $\Re$ with all of these properties. Clearly $v$ can be calculated by using the same methods as those described in Section 4 for finding $\theta$. The only difference is that $P(v) \neq P(2 \phi+\psi)$.

THEOREM 8.1. If $\zeta_{v}>-\frac{1}{2}$, then $\omega=v$. If $\zeta_{v}<-\frac{1}{2}$, then the values of $P(\omega)$ can be found from the possibilities listed in the second column of Table 8.1.

TABLE 8.1

\begin{tabular}{|l|l|}
\hline$P(v)$ & possible values of $P(\omega)$ \\
\hline \hline$P(\phi)$ & $P(\phi), P(\psi), P(\phi+\psi)$ \\
\hline$P(\psi)$ & $P(\psi), P(\phi), P(\phi-\psi)$ \\
\hline$P(\phi-\psi)$ & $P(\psi), P(\phi-\psi)$ \\
\hline$P(\phi+\psi)$ & $P(\phi), P(\phi+\psi)$ \\
\hline
\end{tabular}

Proof. If $\zeta_{v}>-\frac{1}{2}$, then either $\zeta_{\theta}>-\frac{1}{2}$ or $P(\theta)=P(2 \phi+\psi)$. In the first case, $\omega=\theta=v$; in the second case, by Lemma 7.2 we have $\zeta_{\theta}<-\frac{1}{2}$ and $\omega=\phi^{*}=v$. If $\zeta_{v}<-\frac{1}{2}$, then we cannot have $P(\theta)=P(2 \phi+\psi)$ by Lemma 7.3; hence, $v=\theta$ and $\zeta_{\theta}<-\frac{1}{2}$. The possibilities for the puncture of $\omega$ can now be found from Table 7.1.

Note that implicit in this result is the fact that we can run the Voronoi algorithm as given in Section 4 without ever having to consider the $(a, b)=(2,1)$ case. That is, instead of finding $\theta_{g}^{(i)}$ in $\Re_{i}$, we find $v_{g}^{(i)}$ in $\Re_{i}$. We will still find $\varepsilon_{0}$. In fact, $\varepsilon_{0}=\prod_{i=1}^{r} v_{g}^{(i)}$ and $r \leqslant p$.

If $P(\omega)$ is one of $P\left(\gamma_{1}\right), P\left(\gamma_{2}\right), \ldots, P\left(\gamma_{k}\right)$, we need to know how to find $\omega$. As described in Section 4 we find the at most $2 k$ possible values $\gamma_{11}, \gamma_{12}, \gamma_{21}, \gamma_{22}, \ldots, \gamma_{k 1}$, $\gamma_{k 2}$ that $\omega$ could be. After finding the least of these $2 k$ values which exceeds $\theta$, we need only determine whether or not $\left|\gamma^{\prime}\right|<\left|\theta^{\prime}\right|$. If so, $\omega=\gamma$; if not we delete this value of $\gamma$ from among the possible values for $\omega$ and try again. A method of 
determining whether or not $\left|\gamma^{\prime}\right|<\left|\theta^{\prime}\right|$ which involves calculations with integers only can be developed by examining the integer $I=N\left(\theta \gamma^{\prime} \gamma^{\prime \prime}-N(\theta)\right) / N(\theta)$. We have $\left|\gamma^{\prime}\right|<\left|\theta^{\prime}\right|$ if and only if $I<0$.

By using the ideas developed above together with the methods described in [8], a computer program was written to implement the NIVCF algorithm on an AMDAHL 470-V7 computer. To get some idea of how certain aspects of the algorithm behave, we computed all of the distinct lattices $\widetilde{T}_{i}$, where $\widetilde{T}_{1}=\mathscr{R}_{1}=\langle 1, \mu, \nu\rangle$ and $\{1, \mu, \nu\rangle$ is a basis of $\vartheta_{\mathscr{F}}$ for each distinct pure cubic field $\mathscr{F}=\mathcal{2}(\sqrt[3]{D})$ with $D \leqslant 91000$. The total number of lattices found was 325354898. We had $\theta^{\prime}+\theta^{\prime \prime}<-1$ for 112413018 of these. We consider these latter lattices in more detail in Table 8.2.

TABLE 8.2

\begin{tabular}{|l||l|}
\hline puncture of $\theta$, frequency & puncture of $\omega$, frequency \\
\hline \hline$P(\phi), 88139367$ & $P(\phi), 83928591 ; P(\psi), 2759217 ; P(\phi+\psi), 1451559$ \\
\hline$P(\psi), 18584743$ & $P(\phi), 1673460 ; P(\psi), 15267239 ; P(\phi-\psi), 1644044$ \\
\hline$P(\phi-\psi), 4440826$ & $P(\psi), 1123515 ; P(\phi-\psi), 3317311$ \\
\hline$P(\phi+\psi), 1248082$ & $P(\phi), 1248082 ; P(\phi+\psi), 0$ \\
\hline
\end{tabular}

Notice that in most of these cases $\omega=\theta+1$, the exception being the case in which the puncture of $\theta$ is $P(\phi+\psi)$, where we found no instance of $\omega=\theta+1$. There does not appear to be any a priori reason for why $\omega$ should never be $\theta+1$ in this case. However, it must be true that if $\omega=\theta+1$, when $P(\theta)=P(\phi+\psi)$, then $\xi_{\psi}<\frac{1}{2}$ and $\left|\eta_{\psi}\right|<2\left|\eta_{\phi}\right|$. It seems, therefore, that $\omega=\theta+1$ would not occur very frequently in this case. Probably, if we were to continue our calculations beyond the values of $D$ considered here, we would find an instance of $\omega=\theta+1$, just as we finally found a $\theta$ with puncture $P(2 \phi+\psi)$ in Section 4.

We also determined all the distinct $R_{i}$ produced by the VCF when $R_{1}$ is defined above and $36000 \leqslant D \leqslant 64000$. We found that the VCF algorithm found a total of 144092600 distinct $\mathscr{R}_{i}$ and the NIVCF found a total of 108315954 distinct $\mathcal{T}_{i}$ for values of $D$ in the same range. The ratio is .7517. In fact, for most values of $D$ the ratio $\pi / p$ seems to be between .74 and .75 . For example, when $D=252727$, $p=135340$ and $\pi=100911$ for a ratio of .7456 . This value is somewhat larger than the corresponding value of about .69 for the NICF versus the RCF algorithm. When we compared the actual timings on running the VCF and NIVCF for finding a specific $\theta_{n}$, we found that the NIVCF found this $\theta_{n}$ value in about 79 to 80 percent of the time needed by the VCF algorithm. Thus, the computational evidence seems to suggest that wherever one is using the VCF algorithm, one should instead use the faster NIVCF algorithm.

Department of Computer Science

University of Manitoba

Winnipeg, Manitoba, Canada R3T 2N2

1. W. W. ADAMS, "On the relationship between the convergents of the nearest integer and regular continued fractions," Math. Comp., v. 33, 1979, pp. 1321-1331.

2. B. N. Delone \& D. K. FAdDeEv, The Theory of Irrationalities of the Third Degree, Transl. Math. Monos., vol. 10, Amer. Math. Soc., Providence, R. I., 1964. 
3. A. HuRwitz, “Über eine besondere Art der Kettenbruchentwicklung reeller Gröszen," Acta Math., v. 12,1889 , pp. $367-405$.

4. B. Minnigerode, "Uber eine neue Methode, die Pellsche Gleichung aufzulösen,” Gött. Nachr. 1873, 619-653.

5. G. VoronoI, On a Generalization of the Algorithm of Continued Fractions, Doctoral Dissertation, Warsaw, 1896. (Russian)

6. H. C. Williams \& P. Buhr, "Calculation of the regulator of $Q(\sqrt{D})$ by use of the nearest integer continued fraction algorithm,” Math. Comp. v. 33, 1979, pp. 369-381.

7. H. C. Williams, "Some results concerning the nearest integer continued fraction expansion of $\sqrt{D}$," J. Reine Angew. Math., v. 315, 1980, pp. 1-15.

8. H. C. Williams, G. Cormack \& E. Seah, "Calculation of the regulator of a pure cubic field," Math. Comp., v. 34, 1980, pp. 567-611.

9. H. C. Williams, "Improving the speed of calculating the regulator of certain pure cubic fields," Math. Comp., v. 34, 1980, pp. 1423-1434.

10. H. C. Williams, G. W. DuecK \& B. K. Schmid, “A rapid method of evaluating the regulator and class number of a pure cubic field,” Math. Comp., v. 41, 1983, pp. 235-286. 